\title{
Focal Modulation Microscopy: Principle and Techniques
}

\author{
Nanguang Chen, Guangjun Gao and Shau Poh Chong \\ National University of Singapore, \\ Singapore
}

\section{Introduction}

Focal modulation microscopy (FMM) is an emerging single-photon fluorescence microscopy technique that can provide superior image contrast with sub-micron spatial resolutions at large penetration depths in highly scattering media such as biological tissues, mainly by preserving the signal-to-background ratio (SBR). To achieve this, FMM utilizes the coherence property of the light source, through a spatio-temporal modulation scheme to differentially phase modulate segments of the excitation beam. These segments of the beam, when being focused by the objective lens, generate an intensity modulation exclusively at the focal region. Demodulation of the collected fluorescence signal at the designated modulation frequency could allow us to discriminate the in-focus fluorescence from the multiple-scattered background, hence greatly enhance the SBR compared to confocal microscopy. More importantly, the penetration depth of FMM can be significantly improved as the degradation of the image contrast is considerably much slower, and thus could potentially revolutionize the clinical and biomedical applications of FMM for in vivo highresolution visualization of biological specimens. Up to date, a penetration depth up to $600 \mu \mathrm{m}$ has been demonstrated with biological specimens.

In this book chapter, we first highlight the major challenges of optical imaging in achieving large penetration depths when visualizing biological tissues, with further descriptions on the fundamental issues that determine the penetration depths achievable. Then, current optical techniques that are progressively important for deep imaging are briefly reviewed, in which confocal microscopy and two-photon microscopy will be emphasised, mainly due to their superior spatial resolution as well as optical sectioning capability. Distinctive technical differences between these microscopy techniques and advantages of FMM as compared to aforementioned techniques are also being outlined as guidance for readers to adopt the appropriate techniques for their applications.

Subsequently, the core principle of the FMM will be introduced through the concept of point-spread-function (PSF) based on scalar diffraction theory. The performance of FMM imaging in turbid media is also being assessed and validated through our theoretical study that includes scalar diffraction theory and Monte Carlo simulation methods. In particular, the SBR improvement as compared to confocal microscopy is presented. We then discuss the effect of aperture configurations on the modulation depths of FMM, that is crucial in optimizing FMM. 
The next section of this book chapter will cover some of the implementations of FMM since it was invented in year 2008. Notably, two phase modulation schemes - double reflecting mirrors, and FMM based on acousto-optical modulators that are significantly progressive in term of stability and image performance will be described in details. Some of the images acquired using our FMM systems will also be compared side-by-side with confocal images to demonstrate the improvement in image qualities. We have tested on a wide collection of biological specimens, including but not limited to chicken chondrocytes, mouse brain vasculatures, Drosophila fruitfly as well as tissue phantom. Various fluorescence probes are also being utilized with FMM, proving its compatibility for the study of most fluorescencerelated biological parameters.

For a concluding remark, we give a glimpse of future development of FMM, with emphasis on its applications in addressing basic problems in biomedical researches and understanding the pathophysiology of human diseases.

\section{Challenges and potential impacts of deep tissue imaging}

The desire to probe even deeper into biological specimens while resolving microstructural details and observing specific molecular events has grown over the last decade. This is largely because the knowledge obtained by looking at the biological system continuously over the entire entity coupled with functional information, as compared to individual snapshots via tiny-sectioned specimens, could greatly empower the understanding of cellular biology as well as diagnosis and prognosis of many diseases. However, the penetration depth that can be achieved in visualizing biological specimens is usually quite shallow compared to the total tissue volume, and is not absolutely defined mainly because image degradation by background and noise is a gradual process. Here, we briefly overview the current state of deep tissue imaging.

First and foremost, the achievable penetration depth depends largely on the optical properties of the specimen - how transparent, scattering, or opaque it is (Oheim, Beaurepaire et al. 2001). For instance in the case of zebrafish Danio rerio, one can see entirely through an embryo or larva with basic light and fluorescence microscopy and hence zebrafish has become a model organism in developmental genetics and neuroscience (Detrich, Westerfield et al. 2010). Similarly, other organisms that are virtually transparent, such as early-stage roundworm Caenorhabditis elegans and fruit fly Drosophila melanogaster are also popular for the in vivo study of many biological processes. In particular, fruit fly Drosophila melanogaster has been valuable in identifying genes that control embryonic development, as well as the formation of the nervous system. Nevertheless, many other organisms and their body parts are so opaque that it is impossible for the current state-ofarts microscopy techniques to achieve more than $1 \mathrm{~mm}$ penetration depth with uncompromised image quality. As an example, one can resolve little or no cellular details within the cerebral cortex without first drilling holes on the skull of mammals. Very often, this is done with an invasive biocompatible window opened on the skull of a mouse brain to allow an imaging depth up to $600 \mu \mathrm{m}$ (Kleinfeld, Mitra et al. 1998; Svoboda, Helmchen et al. 1999), which corresponds to the cortical layers 1-3 (Theer, Hasan et al. 2003), yet is still very shallow. 


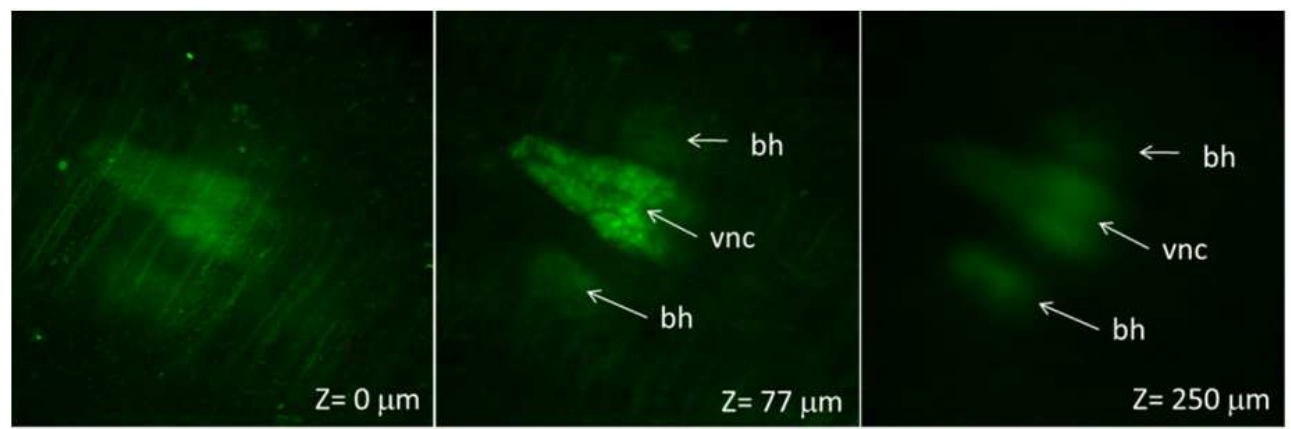

Fig. 1. Image stack of Drosophila pupa expressing elav-GFP to label CNS (brain and ventral nerve cord). The images were acquired with a confocal microscope Zeiss 5 live (10x objective).

Secondly, the imaging depth is also fundamentally limited by the onset of out-of-focus fluorescence generation due to light scattering near the top of the sample (Ying, Liu et al. 1999). At large imaging depths, this out-of-focus background fluorescence would gradually overwhelm the in-focus signal. As a consequence, a loss of image contrast will be observed (Theer, Hasan et al. 2003). Thus, it is generally difficult to preserve the quality of image when imaging at depth more than a few scattering mean free path 1 (MFP). One specific example on how scattering has hindered the study of developmental biology in Drosophila fruitfly model is illustrated in the Fig. 1. It is clearly seen that the blurriness of brain hemispheres (bh) and ventral nerve cord (vnc) in deeper tissue sections $(250 \mu \mathrm{m})$ in contrast to better resolving capability of the confocal microscope at shallower region (i.e. $77 \mu \mathrm{m}$ ). The poor contrast at large penetration depth is due to the attenuation of the fluorescence emission as well as multiple scattering.

This severe limitation of penetration depth of high-resolution optical imaging techniques that can only applied to physically sectioned biological samples through laborious sectioning procedures, or to natural transparent live specimens, such as organisms at very early stages of development, outlined the urgent need to improve in vivo visualization capability in thick biological tissues. For instance, current high-resolution intravital imaging techniques could permit visualization of tumor microstructure and vascular morphology superficially (300 - 400 $\mu \mathrm{m}$ depth), which is over volumetric regions that are a small fraction of the total tumor volume in small animal models (Vakoc, Lanning et al. 2009), thus very much limited our understanding on the tumor microenvironment over multistate and multiscale level.

High-resolution optical techniques with large penetration depth can be proved vital in biomedical applications as they allow observation of enormous biological processes within large tissue volumes especially for in vivo visualization of dynamic phenomenons in developing organism to address some of the basic problems in development biology, such as understanding the development phase of central nervous system in Drosophila fruitfly

${ }^{1}$ The average distance between each scattering event. 
over the whole body volume, as depicted by Fig. 1. Visualization beyond penetration limit of modern microscopy techniques will not only open new pathways in understanding the signaling and interactions of tissues with drugs and environmental factors in adult model organisms but also could potentially useful for noninvasive imaging and spectroscopy of intact biological tissues of human subjects, including but not limited to nondestructive diagnosis of major human diseases such as early cancer detection (Zeng, Vangveravong et al. 2007; Pavlova, Williams et al. 2008). Specifically, the capability to visualize tissue microstructures at penetration depth up to $1 \mathrm{~mm}$ in biological tissues will be clinically important in in vivo diagnosis of skin melanomas in dermatology. We further highlight that deep imaging could also assist in dissecting tumor pathophysiology of intact tumor microenvironment (Jain, Munn et al. 2002; Vakoc, Lanning et al. 2009), as well as understanding how the single cells respond to the damage of the nervous system in neuropathology (Misgeld and Kerschensteiner 2006) of living animal models, just to list a few.

In summary, the development of optical techniques with large penetration depth will have great impacts in revolutionizing the usages of optical techniques in biomedical and clinical applications.

\section{Current optical techniques for deep tissue imaging}

This section gives a brief review of some optical microscopy techniques that are being important for observation of thick biological specimens. The principles of each technique are being described along with their advantages and disadvantages, in an attempt to give a snapshot of current capabilities in deep tissue imaging.

\subsection{Confocal microscopy}

The invention of confocal microscopy (CM) (Minsky 1961; Minsky 1988; Pawley 2006) has radically transformed optical imaging in biology research as it provides diffraction-limited spatial resolution with unprecedented optical sectioning capability to eliminate from images the background caused by out-of-focus light and scatter (Conchello and Lichtman 2005). This is achieved through a small pinhole (point) aperture or single-mode fiber being used in the detection/image plane which acts as a spatial filter/angular gate to block highly scattered out-of-focus light (see Fig. 2). This removal of undesired out-of-focus light not only enhanced the image contrast but more importantly also allowed three-dimensional imaging as images of thin slices can be acquired over thick specimens. The pinhole also rejects the scattered halo of light around the illumination spot if the pinhole size is small enough (i.e. about the size of Airy disk) (Conchello and Lichtman 2005), optimizing the point spread function for good resolution. In other words, the pinhole with appropriate size can effectively ensure that most of the ballistic photons contribute mostly of the signals being collected.

Nonetheless, this selective detection mechanism becomes less effective when the focal points moves deeper into the specimens or thick samples are being used, as multiple scattered stray photons will eventually leak through the pinhole (Kempe, Thon et al. 1994; Schmitt, Knuttel et al. 1994; Kempe, Rudolph et al. 1996), eroding the image contrast and signal-tonoise ratio (Dunn, Smithpeter et al. 1996). Furthermore, in most cases for deep tissue imaging, larger pinhole aperture radius are preferred to allow more photons to be collected 


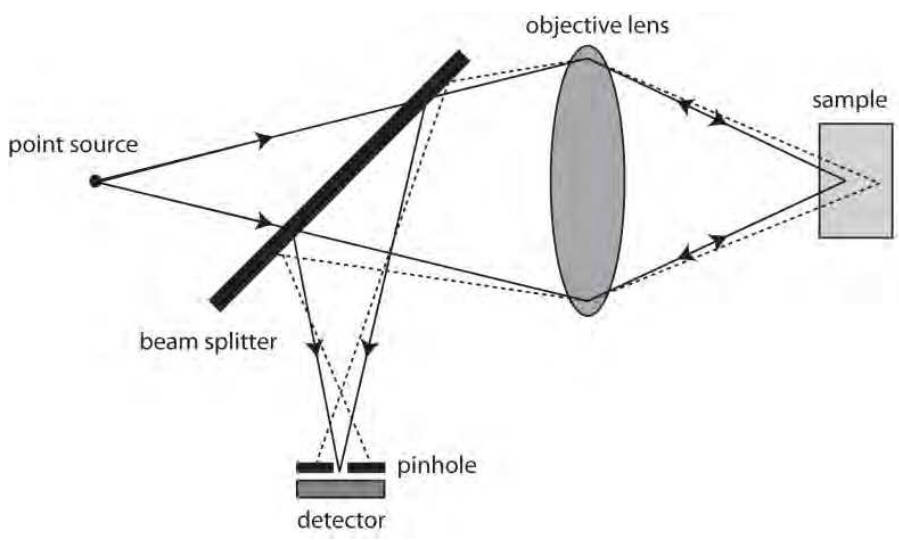

Fig. 2. Schematic diagram for a confocal reflection microscope. Dotted line represents the path for the light coming from out-of-focus areas in the sample; which will be blocked by the pinhole with appropriate size.

to compensate for the signal lost by attenuation, which could further deteriorate the imaging performance. This is because increasing the pinhole aperture size though will marginally improve the signal-to-noise ratio but will also drastically reduce the depth discrimination power (i.e. optical sectioning capability) of the microscope, leading to diminishing image contrast. Hence, very often, it is the trade-off between the signal level and background scattered-light rejection that places a fundamental limit on the sectioning capability of the microscope (Schmitt, Knuttel et al. 1994) as well as the imaging depth (Smithpeter, Dunn et al. 1998; Theer and Denk 2006). As the scattering effects gradually dominate at larger depth, image contrast of CM will eventually lost and thus limit its usefulness at near the tissue surface (less than $100 \mu \mathrm{m}$ ) (Conchello and Lichtman 2005). In fact, imaging of CM in turbid media such as living tissue is usually performed at imaging depth up to few tens of microns only due to limited contrast at higher depth aforementioned.

By combining with other mechanism to reject the multiple-scattered photons (Gan, Schilders et al. 1997), it is possible to improve the penetration depths of CM. Notably, optical coherence microscopy (OCM) combines the coherence gating mechanism of optical coherence tomography (OCT) and the pinhole effect of the CM to enhance the selection of ballistic photons for image reconstruction. Significant improvement in optical sectioning capability and penetration depths has been demonstrated (Izatt, Hee et al. 1994). Nevertheless, OCM is only sensitive to back-scattered light and not applicable for visualization of fluorescence, thus severely limited its biological applications.

In term of spatial resolution, $\mathrm{CM}$ could provide superior lateral $(\approx 1.4 \times$ improvement $)$ and axial $(\approx 1.05 \times$ improvement) resolution compared to conventional widefield microscopy (Sheppard and Wilson 1980; Gu 1996). However, this improvement in spatial resolutions is only appreciable when infinitesimal pinhole is being used which preclude any signal collections. Therefore, optimal pinhole size between $60 \%$ and $80 \%$ of the diameter of the diffraction-limited spot (Sandison, Piston et al. 1995) are generally used, with further adjustment on the pinhole size to accommodate for desired optical sectioning capability and signal level. 


\subsection{Two-photon microscopy}

Another promising optical techniques for subcellular spatial resolution in vivo deep tissue imaging is nonlinear two-photon fluorescence microscopy (Denk, Strickler et al. 1990; Helmchen and Denk 2005; Svoboda and Yasuda 2006). Two-photon fluorescence microscopy is very similar to $\mathrm{CM}$ in term of the system design except that it uses 'higherorder' light-matter interactions to generate fluorescence excitation in which two low-energy photons arrive 'simultaneously' (within $\sim 0.5$ femtoseconds, see Fig. $3 \mathrm{~A}$ ) at a fluorescence molecule combine their energies to promote the molecule to an excited state, which then proceeds along the normal fluorescence-emission pathway.

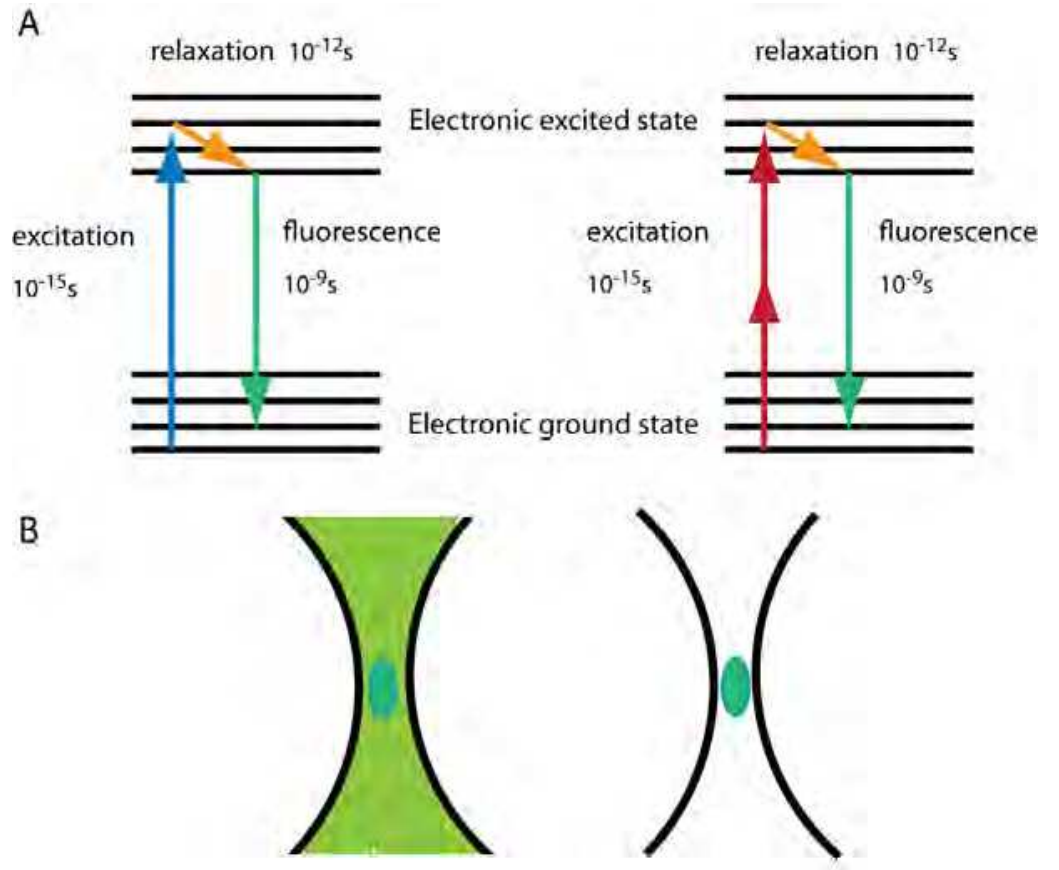

Fig. 3. Single-photon and two-photon process. A. Jablonski diagrams for one-photon excitation and two-photon excitation. B. Schematics of one-photon versus two-photon fluorescence emission.

The process of two-photon absorption requires extremely high concentration of excitation in spatial and temporal domain. Hence, very expensive pulse lasers that emit 'ultrashort' pulses in the range of hundreds femtoseconds with correspondingly high peak intensities have to be used with a high NA objective to focus the light beam into a diffraction-limited volume. A highly enhanced two-photon fluorescence system in focused laser enable vast majority of the fluorescence excitation occurs in a focal volume that can be as small as $\sim 0.1 \mu \mathrm{m}^{3}$. This localization of excitation will limit the out-out-focus fluorescence excitation because even the excitation beam illuminated the sample like the case of CM (i.e. over the illumination cone as illustrated in Fig. 3B), the out-of-focus excitation light are too weak to generate appreciable two-photon fluorescence. Thus, intrinsic optical sectioning and high- 
contrast imaging can be achieved. As all the fluorescence photons are assumed generated from the focal region and can be collected as useful signals for imaging, spatial filters in the detection path (i.e. the detector pinhole in the $\mathrm{CM}$ ) is not necessary, allowing higher collection efficiency (i.e. for greater signal level) and unprecedented imaging depths (Deng and $\mathrm{Gu}$ 2003). In particular, imaging up to a depth of $600 \mu \mathrm{m}$ for vasculature and $700 \mu \mathrm{m}$ for neuron of mouse brain has been achieved with femtosecond mode-locked Titanium: Sapphire (Ti: S) oscillators (Helmchen and Denk 2005).

It is worth to mention that these penetration depths are achieved partly due to the use of near-infrared excitation light that reduces light scattering, however, the achievable lateral and axial resolution are both slightly worse due to longer wavelength light sources are being used. Furthermore, two-photon fluorescence microscope uses very expensive pulse laser that may not be easily affordable for most research groups. Another concern for application of two-photon microscopy is the photo-damage/photobleaching at the focal volume, mainly due to the high intensity pulse laser to elicit fluorescence at the focus point. It is further claimed that the photobleaching that occurs at the focus is higher for twophoton absorptions than it is for single photon absorptions for comparable emission yields (Patterson and Piston 2000; Dittrich and Schwille 2001). This is undesirable for functional imaging as photobleaching at the focus is likely to be more detrimental than bleaching at plane above and below the focus. On top of that, the optical indicators that can be used with two-photon microscopy are only a small subset of the indicators that can be used with single-photon excitation microscopy, mainly due to the limited availability of fluorescence probes with large "two-photon absorption cross-section". This limitation could severely constrain the types of biological parameters and processes that can be studied using twophoton fluorescence microscopy.

Regardless of the superiority of nonlinear mechanism to inhibit the generation of out-offocus fluorescence, the performance of two-photon microscopy could still be affected by multiple scattering. At extended depths, the ability of excitation light to reach the focus unscattered (ballistic excitation photons) and of emitted fluorescence to arrive at the detector, with generation of out-of-focus fluorescence near the surface, are placing a fundamental limit on how deep two-photon microscopy can probe (Theer and Denk 2006; Kobat, Durst et al. 2009). Recent efforts on improving the penetration depths of two-photon microscopy by using longer wavelength excitation (Kobat, Durst et al. 2009) or by reducing the pulse width of excitation laser (Theer, Hasan et al. 2003) (to increase two-photon fluorescence excitation yield) has been very successful with imaging depths over $1000 \mu \mathrm{m}$ being reported (Theer, Hasan et al. 2003; Kobat, Durst et al. 2009). Still, those techniques are very selective on the availability of fluorescence probes that can be excited using nearinfrared light and are still very technically demanding (Kobat, Durst et al. 2009) to be adopted by most biological research groups.

\subsection{Optical coherence tomography}

Coherence optical tomography (OCT) (Huang, Swanson et al. 1991; Fujimoto 2003) is an emerging biomedical optical imaging technique that is capable of considerable high resolution and cross-sectional tomographic imaging of microstructures in biological systems. Typically, OCT can provide micron-range axial resolution for an imaging depth up to $2-3 \mathrm{~mm}$ in tissue depending on the optical scattering (Fujimoto, Brezinski et al. 1995). 


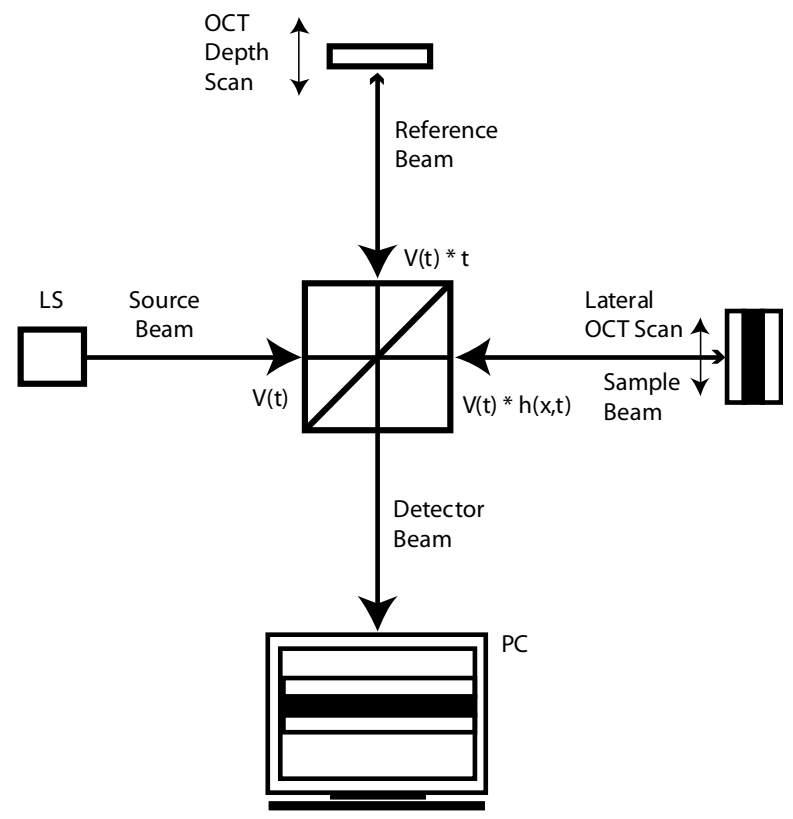

Fig. 4. Standard OCT scheme based on a low time-coherence Michelson interferometer. The intensity $I_{E}$ of the interferometer exit depends on the sample response $h(x, z)$ convolved with the source coherence function $\Gamma_{\text {source }}(z)$. LS $=$ low time-coherence light source; PC = personal computer. Adapted from (Fercher, Drexler et al. 2003).

OCT uses coherence gating (i.e. interference between the back-scattered light and a reference beam) to improve the detection of backscattered light for image reconstruction. OCT can generate good contrasts as only the backscattered light that has a well-defined optical path length and polarization state will interfere constructively with the reference beam to generate strong fringe signals for image reconstruction (see Fig. 4) (Fercher, Drexler et al. 2003). The intensity of the interferometer exit $I_{E}$ can be represented by:

$$
I_{E}(x, z)=I_{s}+I_{R}+2 \operatorname{Re}\left[\Gamma_{\text {source }}(z) \times h(x, z)\right]
$$

where $I_{S}$ and $I_{R}$ are the intensity at the sample and reference arms respectively, $\Gamma_{\text {source }}$ being the source coherence function and $h(x, z)$ is the sample response function (i.e. determined by the sample geometry and reflectivity due to refractive index mismatches).

Due to its relatively larger penetration depths, OCT has found applications in ophthalmology and dermatology (Prati, Regar et al. 2010). Specifically, OCT has been heavily applied for diagnosis of various eye diseases such as age-related macular degeneration and glaucoma (Fercher 2010; Prati, Regar et al. 2010) On top of that, OCT has also been applied in functional imaging such as spectroscopic imaging of tissue properties, Doppler blood flow measurement and quantification of blood oxygenation or tissue birefringence (Fujimoto 2003). Recently, second generation OCT, termed optical frequency domain imaging (OFDI) (Vakoc, Lanning et al. 2009) has been very successful for the understanding of biological parameters of the tumor microenvironment in vivo. 
Although substantially high-resolution at imaging depth up to $3 \mathrm{~mm}$ is readily achievable using OCT, its lack of molecular contrast especially incompatibility with fluorescence microscopy has restricted its applications for the study of many other biological processes.

\subsection{Photo-acoustic tomography}

Photo-acoustic tomography (PAT) (Wang, Xu et al. 2002; Wang, Ku et al. 2004), being one of the fastest-growing area of biomedical imaging technology over the last decade, offers highresolution three-dimensional imaging with rich optical contrast in vivo at depths beyond the optical transport mean free path (i.e. $1 \mathrm{~mm}$ in human skin) (Wang 2009). In the photoacoustic phenomenon, excitation light (short-pulsed laser is normally used for efficient ultrasound generation) is absorbed by a material and converted to heat, and the subsequent thermoelastic expansion generates an acoustic wave. This acoustic wave can then be detected by using an ultrasonic detector for the reconstruction of ultrasound image. In fluorescence mode, ultrasonic detection of pressure waves generated by the absorption of pulse light by fluorescence proteins in elastic media enable high-resolution visualization of fluorescent proteins few millimetres deep within highly light-scattering living organisms.

PAT combines the optical imaging and ultrasound imaging, enabling rich optical contrast and high ultrasonic resolution imaging modality. Typically, the ultrasonic scattering coefficient in biological tissue is $2-3$ orders of magnitude less than the optical equivalent. Hence, PAT can detect the ultrasonic waves even at a depth of multiple folds larger compared to existing high-resolution optical imaging techniques, such as confocal microscopy and two-photon microscopy, thus enabling deeply penetrating functional (Wang, Pang et al. 2003; Yang, Xing et al. 2007) and molecular (Li, Zemp et al. 2007; Li, Oh et al. 2008) imaging in biological samples. For instance, highly optimized PAT could enable visualization of fluorescent proteins few millimeters deep within highly light-scattering living organisms.

Nevertheless, the in-plane spatial resolution of the photo-acoustic images achievable is fundamentally limited by the effective bandwidth of the ultrasonic detector $(20 \mathrm{MHz})$, leading to $\sim 38 \mathrm{um}$ diffraction limited resolution and even worse for axial resolution (Razansky, Distel et al. 2009). Recent higher lateral resolution around $5 \mathrm{um}$ has been reported but at a much shallower penetration depth of $0.7 \mathrm{~mm}$ (Wang 2009), which is not better than current techniques such as two-photon microscopy. Furthermore, very often the specimens have to be immersed in acoustic medium for better coupling of the propagation of acoustic wave to the ultrasonic detector, which might not be suitable for all types of living biological specimens.

\subsection{Others recent developments}

Another very promising technique, termed single plane illumination microscopy (SPIM) (Huisken, Swoger et al. 2004) or light-sheet-based fluorescence microscopy (Keller, Schmidt et al. 2010) in which a single plane in specimen is quickly scanned with a thin laser beam while a 2D camera-based detection arm records a fluorescence image at a right-angle to the illumination axis. Light-sheet-based microscopy could greatly enhance the image acquisition speed and the image contrast while limited the photobleaching and phototoxicity on the specimens (Keller, Schmidt et al. 2008; Keller and Stelzer 2008). However, this technique 
usually requires dedicated image post-processing to improve the spatial resolutions (for instance, deconvolution) (Verveer, Swoger et al. 2007; Keller, Schmidt et al. 2010) and no penetration depths over $100 \mu m$ have been reported up to date.

Other high-resolution optical imaging techniques such as 4-Pi (Hell, Stelzer et al. 1994) and I5M (Gustafsson, Agard et al. 1999) microscopy based on coherent collection of fluorescence using a pair of diametrically opposed objectives which could enhance the axial resolution by a factor of 3-7 along the optical axis; far-field optical microscopy such as stimulated emission depletion (STED) microscopy (Dyba and Hell 2002) uses superlocalized depletion of the excited state by stimulated emission with few tens nanometers spatial resolution being demonstrated (probably the highest resolution using optical techniques for living cells) and total internal reflection fluorescence microscopy (TIRF) (Axelrod 2003) that uses decaying evanescent wave are all severely limited in penetration depth and thus not extensively reviewed here.

\subsection{Summary}

There is a considerable large gap in the development for optical microscopy techniques that can achieve high penetration depth with subcellular spatial resolution, for vast applications in biomedical researches. Table 1 summarizes the strengths and limitations of some of the imaging modalities for subcellular-resolution deep imaging being described in this book chapter. Therefore we developed Focal Modulation Microscopy (FMM) (Chen, Wong et al. 2008), a novel technique that aims for imaging depth greater than but not limited to $500 \mathrm{um}$ with subcellular spatial resolution and molecular specificity. The principle of FMM will be further elaborated in the next section.

\begin{tabular}{lll}
\hline Embodiments & Advantages & Limitations \\
\hline $\begin{array}{l}\text { Confocal } \\
\text { Fluorescence }\end{array}$ & $\begin{array}{l}\text { High-resolution with optical sectioning. } \\
\text { Much cheaper laser source than two- } \\
\text { photon imaging. }\end{array}$ & $\begin{array}{l}\text { Shallow penetration depths } \\
\text { due to multiple scattering } \\
\text { effects. }\end{array}$ \\
\hline $\begin{array}{l}\text { Two-Photon } \\
\text { Fluorescence } \\
\text { Microscopy }\end{array}$ & $\begin{array}{l}\text { Optical sectioning. Reduced } \\
\text { photobleaching and photodamage with } \\
\text { large penetration depths up to 1 } \mathrm{mm} .\end{array}$ & $\begin{array}{l}\text { High cost of ultrashort- } \\
\text { pulsed laser and limited } \\
\text { option of fluorescence } \\
\text { probes. }\end{array}$ \\
\hline $\begin{array}{l}\text { Optical } \\
\text { Coherence }\end{array}$ & $\begin{array}{l}\text { Large penetration depth up to } 3 \mathrm{~mm} \text { and } \\
\text { ease of implementation. }\end{array}$ & $\begin{array}{l}\text { Lack of molecular contrast } \\
\text { due to incompatibility with } \\
\text { fluorescence imaging and } \\
\text { high level of speckle noise. }\end{array}$ \\
\hline $\begin{array}{l}\text { Photoacoustic } \\
\text { Tomography }\end{array}$ & $\begin{array}{l}\text { Good penetration depths up to few mm } \\
\text { and compatible with fluorescence }\end{array}$ & $\begin{array}{l}\text { Poorer spatial resolutions } \\
\text { than confocal and two- } \\
\text { photon microscopy. }\end{array}$ \\
\hline $\begin{array}{l}\text { Focal } \\
\text { imaduing. }\end{array}$ & $\begin{array}{l}\text { Optical sectioning. Better penetration } \\
\text { depths than confocal microscopy using } \\
\text { Microscopy }\end{array}$ & $\begin{array}{l}\text { Photobleaching (same as } \\
\text { confocal microscopy). }\end{array}$ \\
& in fluorescence and reflectance mode. & \\
\hline
\end{tabular}

Table 1. A comparison of optical imaging modalities with good resolution and penetration depths. 


\section{Principle of focal modulation microscopy}

Focal modulation microscopy (FMM) (Chen, Wong et al. 2008; Wong, Chong et al. 2009) is a novel optical microscopy technique based on single photon excited fluorescence. It can provide sub-micron spatial resolution at large penetration depth in living tissues mainly by preserving the SBR of the image. In FMM, a phase modulator is employed to sinusoidally phase modulate (or frequency shifting) half of the spatial extent of the excitation beam. As the beam being tightly focused by the objective lens, interference of the both half-beams generates an intensity modulation at the designated frequency around the focal volume. This modulated excitation focus thus results in a modulated fluorescence signal from the focal volume. Although both the ballistic and scattered photons could reach the focal plane, only the ballistic photons contribute to the oscillatory excitation, as they have well-defined phase and polarization. Therefore, in principle, "out-of-focus" fluorescence emission should not be modulated as they are originated from scattered excitation. Subsequently, a simple lock-in technique can be implemented to retrieve the fluorescence signal excited by ballistic light and to reject the contribution from diffusive-light-excited fluorescence.

\subsection{Excitation beam modulation}

Here we present the principle of FMM in mathematical model. As FMM is a technique that employs a spatiotemporal phase modulator to sinusoidally modulate a spatial fraction of the excitation beam, we can define the excitation field:

$$
E_{i}\left(r_{i}\right)=h_{i 1}\left(r_{i}\right)+h_{i 2}\left(r_{i}\right) e^{i \varphi(t)}
$$

in which $r_{i}$ is the spatial coordinates and the $h_{i 2} e^{i \varphi(t)}$ term describe the fraction of the excitation field being phase modulated with a periodic function $\varphi(t)$ (at the frequency $f$ ) in contrast to the unmodulated fraction $h_{i 1}$. The corresponding excitation intensity around the focal spot is thus given by:

$$
I_{i}\left(r_{i}\right)=\left|E_{i}\left(r_{i}\right)\right|^{2}=E_{i}\left(r_{i}\right) \times E_{i}^{*}\left(r_{i}\right)=\left(h_{i 1}+h_{i 2} e^{i \varphi(t)}\right) \times\left(h_{i 1}+h_{i 2} e^{i \varphi(t)}\right)^{*}
$$

Expanding the Eqn. 3, we obtained:

$$
\begin{gathered}
I_{i}\left(r_{i}\right)=h_{i 1}\left[h_{i 1}\right]^{*}+h_{i 2} e^{i \varphi(t)}\left[h_{i 2} e^{i \varphi(t)}\right]^{*}+h_{i 1}\left[h_{i 2} e^{i \varphi(t)}\right]^{*}+h_{i 2} e^{i \varphi(t)}\left[h_{i 1}\right]^{*}=\left|h_{i 1}\right|^{2}+\left|h_{i 2}\right|^{2}+ \\
2 \operatorname{Re}\left(h_{i 1} h_{i 2}^{*} e^{i \varphi(t)}\right)=\left|h_{i 1}\right|^{2}+\left|h_{i 2}\right|^{2}+2\left|h_{i 1} h_{i 2}^{*}\right| \cos \left(\varphi(t)+\arg \left(h_{i 1} h_{i 2}^{*}\right)\right)
\end{gathered}
$$

We could observe from the last line of Eqn. 4 that the first two terms are the fraction of excitation intensity which is unchanged over time whereas the last term corresponds to oscillatory intensity at the modulation frequency $f$. In a FMM system, the unmodulated field $h_{i 1}\left(r_{i}\right)$ and the modulated field $h_{i 2}\left(r_{i}\right)$ are generally spatially separated, due to spatiotemporal phase modulation at the objective aperture. The excitation intensity is therefore a constant except at locations around the focal point, in which there is a significant overlapping between $h_{i 1}\left(r_{i}\right)$ and $h_{i 2}\left(r_{i}\right)$. At the focal point, $h_{i 1}\left(r_{i}\right)$ and $h_{i 2}\left(r_{i}\right)$ are usually in phase so that $\arg \left(h_{i 1} h_{i 2}^{*}\right)=0$. The maximal excitation intensity is reached when $\cos (\varphi(t))=$ 1 (in phase),

$$
I_{\text {in-phase }}=\left|h_{i 1}\right|^{2}+\left|h_{i 2}\right|^{2}+2\left|h_{i 1} h_{i 2}^{*}\right|
$$

The minimal excitation intensity is : 


$$
I_{o f f-p h a s e}=\left|h_{i 1}\right|^{2}+\left|h_{i 2}\right|^{2}-2\left|h_{i 1} h_{i 2}^{*}\right|
$$

which corresponds to $\cos (\varphi(t))=-1$. The difference between the in-phase and off-phase intensities provides the measure of peak-to-peak value of intensity modulation at the focal point.

\subsection{Excitation point spread function of FMM}

We can find the illumination intensity point spread function (PSF) of the FMM using the scalar diffraction theory. For an aberration-free lens of circular aperture, the illumination intensity distribution is given by (Gu 2000):

$$
I_{\text {illumination }}(v, u)=\left|2 \int_{0}^{1} P(\rho) J_{0}(v \rho) e^{\frac{i u \rho^{2}}{2}} \rho d \rho\right|^{2}
$$

in which $J_{0}$ denotes the zero-order Bessel function and $v=k(N A) r$ and $u=4 k\left[\sin \frac{\alpha}{2}\right]^{2} z$ are radial and axial normalized optical coordinates with the wavenumber $k=2 \pi / \lambda$. The excitation beam is split into two fractions. Let's consider an annular aperture as an example. The excitation beam passing through the outer ring of the annular aperture is subject to a phase-shift varying from 0 to $\pi$ with respect to the excitation beam passed through the inner circle. Hence, the pupil function $P(\rho)$ that describes the field distribution at the lens aperture can be described as:

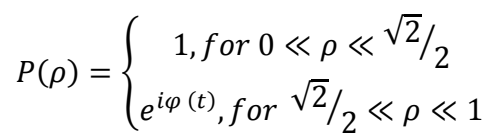

where $\rho$ is the normalized aperture diameter of the objective lens.
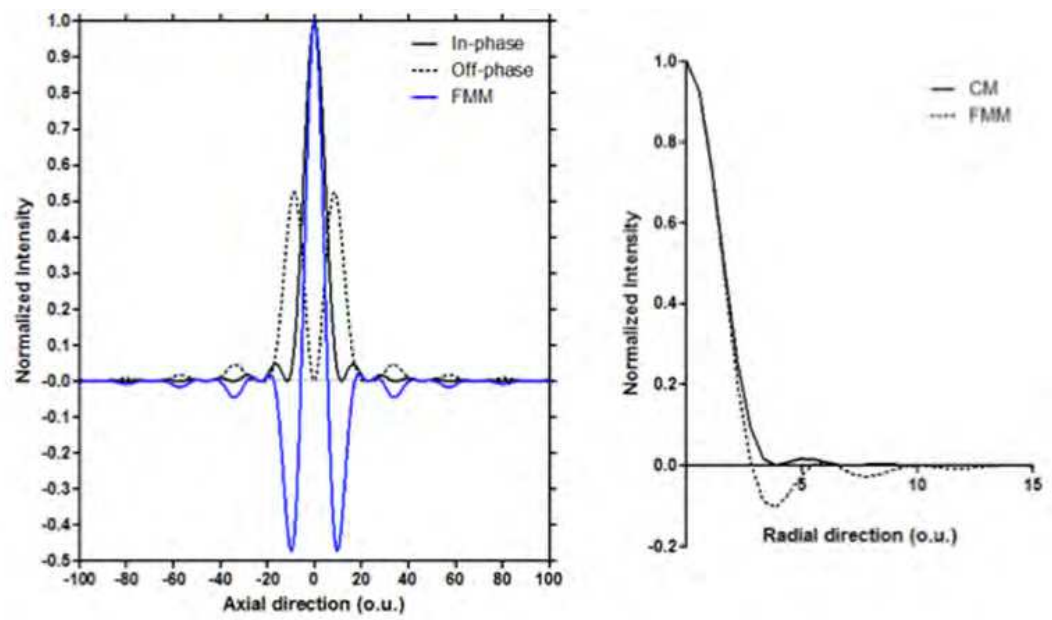

Fig. 5. (Left) Simulated excitation intensity profile along the axial direction of the in-phase, out-phase and FMM signals based on scalar diffraction theory. (Right) Simulated excitation intensity profile along lateral direction of the CM and FMM. 
When the phase profile over the aperture is uniform (i.e. $\varphi(t)=0$ ), the intensity distribution along the optical axis is identical to the illumination point spread function of $\mathrm{CM}$; on the other hand, when $\varphi(t)=\pi$, there will be a strong destructive interference of the beams at the focal spot, leading to the off-phase intensity profile (dashed) as shown in Fig. 5 (Left). The difference between these two excitation intensity profiles is the effective illumination point spread function in FMM.

The excitation intensity distribution of the CM and FMM along the lateral direction is plot in Fig. 5 (Right). It is worth to mention that due to the differential mechanism of FMM, there are negative sidelobes in the excitation IPSF of the FMM, albeit with relatively smaller magnitude compared to that of lateral IPSF. In order to extract the FMM signal, an aperture pinhole of size slightly smaller that the central lobe is to be used, in order to exclude the negative side lobes being included in the retrieved signal. These negative side lobes, if being included in the detected signal, will dramatically reduce the FMM signal.

\subsection{Signal-to-background ratio in FMM}

While the basic principle of FMM is straightforward and its performance has been experimentally demonstrated (Chen, Wong et al. 2008), it is desirable to conduct theoretical studies to gain further insight into this technique. In this section, we present a theoretical model that combines the scalar diffraction theory ( $\mathrm{Gu} 2000)$ with Monte Carlo simulation (Schmitt, Knuttel et al. 1994), in which the dependence of SBR on penetration depth are examined and compared for both FMM and CM.

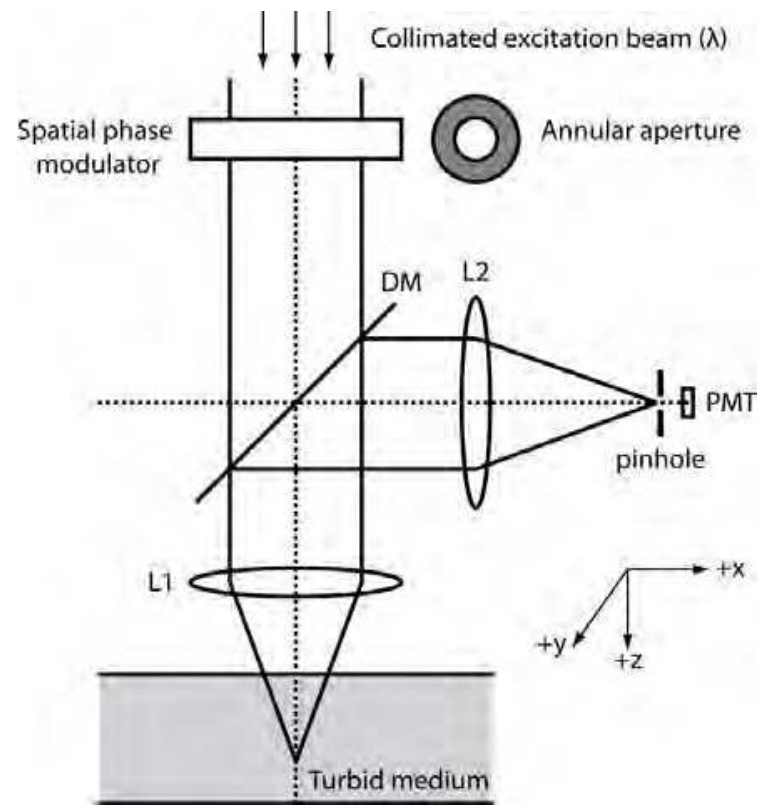

Fig. 6. Model geometry of a FMM/CM setup. Annular aperture with (shaded region) and without phase modulation is studied. L1 and L2 are lens with focal length $\mathbf{9} \mathbf{m m}$. DM is the dichroic mirror and PMT is the photomultiplier tube. 


\subsubsection{Model geometry}

We consider a system geometry shown in Fig. 6. The collimated excitation laser beam is focused by the objective lens L1 to a point located in a slab of turbid medium. The refractive indices are assumed to be matched across the medium surface. The system includes lenses L1 and L2 (Diameter $D=10 \mathrm{~mm}$, focal length $9 \mathrm{~mm}$ ), a dichroic mirror (DM), a pinhole $0.8 \mu \mathrm{m}$ in diameter, a spatiotemporal phase modulator, and a PMT. The optical properties (absorption coefficient $\mu_{a}=0.1 \mathrm{~cm}^{-1}$, scattering coefficient $\mu_{s}=100 \mathrm{~cm}^{-1}$, anisotropy factor $g=0.9$ ) of the turbid medium are typical for biological soft tissues. Fluorescent molecules are homogeneously distributed and are the only absorptive substance in the medium.

\subsubsection{Excitation rate for confocal microscopy}

The excitation light, once entering the turbid medium, is separated into a ballistic component and a scattered component due to scattering events. The fluorescence excitation rate for $\mathrm{CM}$ consists of ballistic (CMB) and scattered (CMS) components:

$$
E_{C M}\left(r_{F}, r_{M}\right)=E_{C M B}\left(r_{F}, r_{M}\right)+E_{C M S}\left(r_{F}, r_{M}\right)
$$

where $r_{F}=\left(x_{F}, y_{F}, z_{F}\right)$ and $r_{M}=\left(x_{M}, y_{M}, z_{M}\right)$ are the locations of the focal point and fluorescent molecules, respectively. The scalar diffraction theory is used to calculate the ballistic excitation rate (in polar coordinates):

$$
E_{C M B}\left(r_{F}, r_{M}\right)=e^{-\mu_{t} z_{M}} \mu_{a} C\left|\int_{0}^{1} J_{0}(v \rho) e^{i u^{\frac{\rho^{2}}{2}}} \rho d \rho\right|^{2}
$$

Here $J_{o}$ denotes the zero-order Bessel function. The normalized optical coordinates $v=$ $k N A \rho$ and $u=4 k \Delta z \sin ^{2}\left(\frac{\alpha}{2}\right)$ are related to the normalized radius $\rho=\sqrt{\left(x_{F}-x_{M}\right)^{2}+\left(y_{F}-y_{M}\right)^{2}}$ and $\Delta z=z_{M}-z_{F}, k$ is the wave number, and the numerical aperture $N A=\sin \alpha=\frac{a}{f}$, with $a$ being the radius of the diffraction aperture at the plane of the objective lens. The constant $C$ is used to normalize the total excitation power to unity. Attenuation of the ballistic component depends on the extinction coefficient $\mu_{t}=\mu_{a}+\mu_{s}$ and the penetration depth $z_{M}$.

Monte Carlo simulation (Schmitt, Knuttel et al. 1994) is employed to obtain the scattered excitation rate $E_{C M S}\left(r_{F}, r_{M}\right)$. Given the focal point $\left(0,0, z_{F}\right)$, photon packets with an initial weight of one are launched at the air-turbid medium interface $(z=0)$. Their initial position and direction are randomly assigned with the probability density function satisfying the following conditions: a) The excitation beam intensity is uniform over the aperture of L1, and b) the photon packets would converge to the focal point if there is no scattering. Then the trajectory of each photon packet is randomly generated following the standard propagation and interaction approach. The weight of a photon packet is adjusted after each interaction:

$$
w_{\text {new }}=w_{\text {old }}-\left(\frac{\mu_{a}}{\mu_{t}}\right) w_{\text {old }}
$$

At the same time, the 'lost' weight is deposited into that position grid as considered as being absorbed by the fluorescence molecules. A simulated trajectory ends when the photon 
escape from the slab surface or the weight drops below a preset threshold. A large number $\left(10^{7}\right)$ of photons are traced to find the average excitation rate $E_{C M S}\left(r_{F}, r_{M}\right)$ for each focal depth. The contribution from the first interaction is attributed to ballistic light and thus excluded from $E_{C M S}\left(r_{F}, r_{M}\right)$.

\subsubsection{Excitation rate for focal modulation microscopy}

For FMM, the equivalent excitation rate is associated with the ballistic light only:

$$
\begin{gathered}
E_{F M M}\left(r_{F}, r_{M}\right)= \\
e^{-\mu_{t} z_{M}} \mu_{a} C\left\{\left|\int_{0}^{1} J_{0}(v \rho) e^{i u \frac{\rho^{2}}{2}} \rho d \rho\right|^{2}-\left|\int_{0}^{0.707} J_{0}(v \rho) e^{i u \frac{\rho^{2}}{2}} \rho d \rho-\int_{0.701}^{1} J_{0}(v \rho) e^{i u \frac{\rho^{2}}{2}} \rho d \rho\right|^{2}\right\}
\end{gathered}
$$

The first term in the above equation (i.e. enclosed in first modulus function) is the excitation intensity when $\varphi(t)=0$ and the second term corresponds to excitation intensity when $\varphi(t)=\pi$.

In Fig. 7 , the excitation rates are compared for an imaging depth of $400 \mu m$. Fig. 7 (a) shows FMM excitation rate (absolution value) as a function of the radial displacement and defocus, while Fig. 7 (b) is the ballistic excitation rate in the CM setup. They are highly concentrated around the focal point and decay rapidly outside of the focal volume. Such a behaviour is desirable for optimal optical sectioning and high-resolution imaging. On the contrary, the diffusive excitation rate for the CM setup (Fig. 7 (c)) is distributed in a rather large volume, although the peak value is about 2 orders of magnitude smaller than that of ballistic excitation rate.

(a)

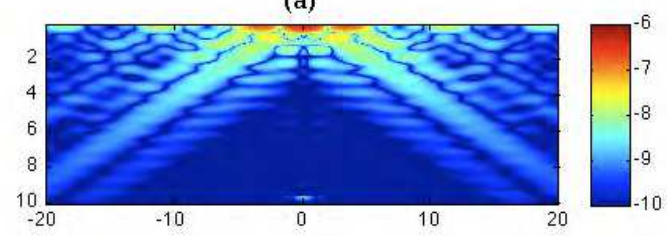

(b)

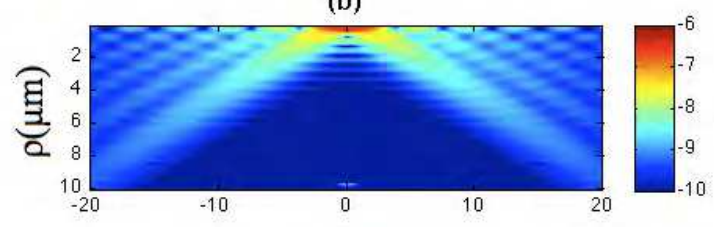

(c)

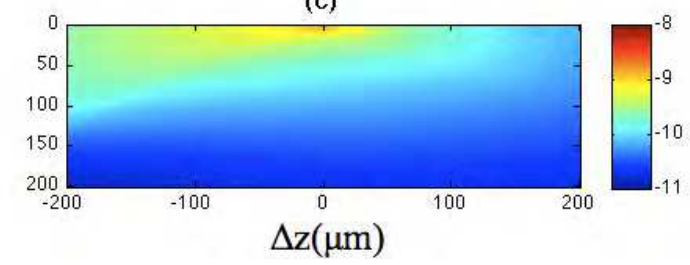

Fig. 7. Ballistic and diffusive excitation rates for an imaging depth of $\mathbf{4 0 0} \boldsymbol{\mu m}$. 


\subsubsection{Detection point-spread-function}

As CM and FMM have the same detection optics, their fluorescence detection efficiencies are identical. For the ballistic fluorescence light, the detection efficiency is given by:

$$
D_{B}\left(r_{F}, r_{M}\right)=\frac{1-\cos \theta}{2} e^{-\mu_{t} z_{M}} C \iint_{S} d x d y\left|\int_{0}^{1} J_{0}\left(v^{\prime} \rho\right) e^{i u \prime^{\frac{\rho^{2}}{2}}} \rho d \rho\right|^{2}
$$

where the two-dimensional integration is over $S$, the image of the pinhole in the medium and centered at $r_{F}$. The optical coordinates $v^{\prime}$ and $u^{\prime}$ are normalized radial and axial distances between $r_{M}$ and the point $\left(x, y, z_{M}\right)$ within $S$. The first term of Eqn. $13, \frac{1-\cos \theta}{2}$ is the solid angles of the detection cone where $\theta$ is half of the angular aperture of L1.

Scattered fluorescence photons are again simulated by the use of Monte Carlo method. Photon packets are launched from a point $\left(0,0, z_{M}\right)$ with the initial directions uniformly sampled. Trajectory of propagation and scattering steps for each photon is generated using the standard Monte Carlo approaches. Photons emerging from the surface without scattering are discarded. Scattered photons, once crossed the upper medium surface, are traced using geometric optics. In order to enhance the simulation efficiency by reusing the trajectories, we actually deploy an array of detectors after L2. The detector array consists of 101 planes along the optical axis, corresponding to focal depths sampled every $10 \mu \mathrm{m}$ from 0 to $1000 \mu \mathrm{m}$ in the medium. Each detection plane has a sensing area $1 \mathrm{~mm}$ in diameter and it is divided into a grid of $1 \mu \mathrm{m}$ by $1 \mu \mathrm{m}$ pixels. A photon captured by the pixel at $r_{\text {det }}$ adds a normalized weight to the scattered detection efficiency:

$$
D_{S}\left(r_{F}, r_{M}\right)=D_{S}\left(r_{F}, r_{M}\right)+\frac{S_{P} W}{N}
$$

Here $W$ is the weight of the photon packet, $S_{P}$ is the pinhole area in $\mu m^{2}$ and $N$ is the total number of photons launched, and $r_{F}$ is the conjugate point of $r_{d e t}$ in the medium.
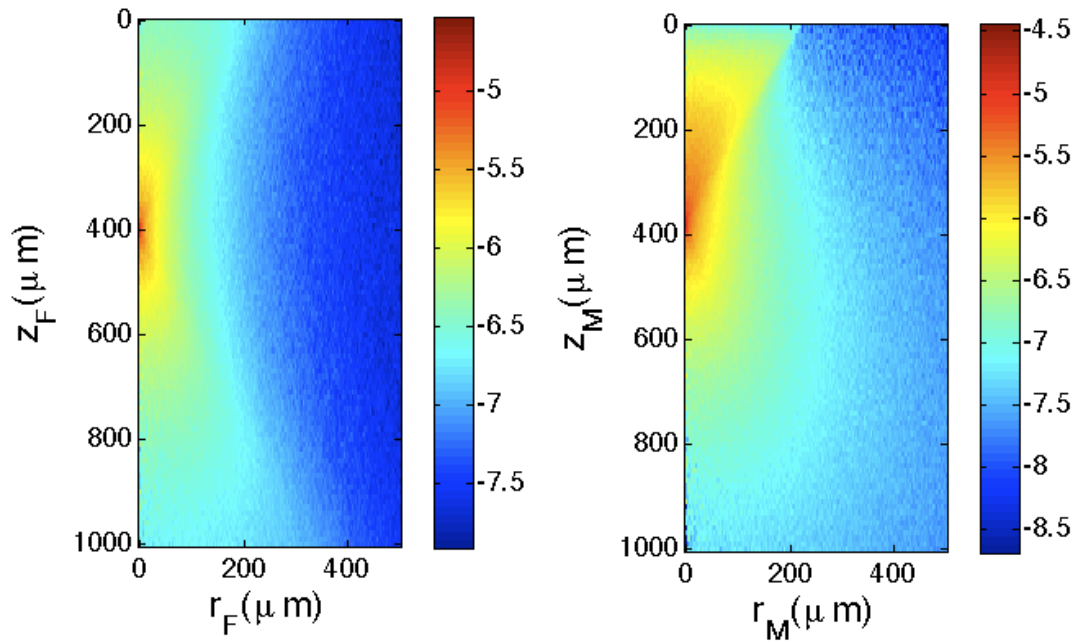

Fig. 8. Scattered detection efficiency as a function of (a) focus point or (b) molecular target position. 
Shown in Fig. 8 (a) is $D_{S}\left(r_{F}, r_{M}=(0,0,400 \mu m)\right)$ as a function of $r_{F}$ and $z_{F}$, obtained with $10^{6}$ simulated photons. The simulation is repeated for the emitter depth range from 0 to $1 \mathrm{~mm}$, at an incremental step of $10 \mu \mathrm{m}$. Then the results are combined to generate the spatial distribution as a function of $r_{M}$ and $z_{M}$, for any given focal depth $z_{F}$. Shown in Fig 8 (b) is $D_{S}\left(r_{F}=(0,0,400 \mu m), r_{M}\right)$, the scattered detection efficiency when the focal point is $400 \mu m$ deep.

\subsubsection{Formulation for signal-to-background ratio}

When the excitation and detection optics are focused at $r_{F}$, the total CM signal can be expressed as a sum of four terms:

$$
\begin{gathered}
I_{C M}\left(r_{F}\right)=\iiint d r_{M}\left\{E_{C M B}\left(r_{F}, r_{M}\right)+E_{C M S}\left(r_{F}, r_{M}\right)\right\} \times\left\{D_{B}\left(r_{F}, r_{M}\right)+D_{S}\left(r_{F}, r_{M}\right)\right\}=I_{C M}^{B B}\left(r_{F}\right)+ \\
I_{C M}^{S B}\left(r_{F}\right)+I_{C M}^{B S}\left(r_{F}\right)+I_{C M}^{S S}\left(r_{F}\right)
\end{gathered}
$$

Here $I_{C M}^{B B}\left(r_{F}\right)=\iiint d r_{M} E_{C M B}\left(r_{F}, r_{M}\right) \times D_{B}\left(r_{F}, r_{M}\right)$ is related to ballistic excitation and ballistic fluorescence photons, and is the desired signal. All the three-dimensional integrals are over the entire volume of the turbid medium. The other three terms contribute to a background $I_{C M}^{B K}=I_{C M}^{S B}+I_{C M}^{B S}+I_{C M}^{S S}$, that is related to scattered photons. The signal to background ratio $(\mathrm{SBR})$ is thus defined as:

$$
S B R_{C M}=\frac{I_{C M}^{B B}}{I_{C M}^{B K}}
$$

in which

$$
\begin{gathered}
I_{C M}^{B K}=\iiint d r_{M}\left\{E_{C M B}\left(r_{F}, r_{M}\right) D_{S}\left(r_{F}, r_{M}\right)+E_{C M S}\left(r_{F}, r_{M}\right) D_{B}\left(r_{F}, r_{M}\right)+E_{C M S}\left(r_{F}, r_{M}\right) D_{S}\left(r_{F}, r_{M}\right)\right\}= \\
I_{C M}^{S B}+I_{C M}^{B S}+I_{C M}^{S S}
\end{gathered}
$$

The total FMM signal is given by

$$
\begin{gathered}
I_{F M M}\left(r_{F}\right)=\iiint d r_{M}\left\{E_{F M M}\left(r_{F}, r_{M}\right) D_{B}\left(r_{F}, r_{M}\right)+E_{F M M}\left(r_{F}, r_{M}\right) D_{S}\left(r_{F}, r_{M}\right)\right\}= \\
I_{F M M}^{B B}\left(r_{F}\right)+I_{F M M}^{B S}\left(r_{F}\right)
\end{gathered}
$$

while the signal to background ratio is simply

$$
S B R_{F M M}=\frac{I_{F M M}^{B B}}{I_{F M M}^{B S}}=\frac{\iiint d r_{M} E_{F M M}\left(r_{F}, r_{M}\right) D_{B}\left(r_{F}, r_{M}\right)}{\iiint d r_{M} E_{F M M}\left(r_{F}, r_{M}\right) D_{S}\left(r_{F}, r_{M}\right)}
$$

\subsubsection{Discussions and summary}

Various CM and FMM signals are compared in Fig. 9 (a). $I_{C M}^{B B}$ and $I_{F M M}^{B B}$ decay exponentially with the focal depth as expected. $I_{F M M}^{B B}$ is slightly weaker than $I_{C M}^{B B}$ because the modulation depth is always less than one. The CM background is dominated by $I_{C M}^{S S}$, which is related to scattered excitation and scattered emission photons. It overwhelms $I_{C M}^{B B}$ for imaging depths over $200 \mu \mathrm{m}$. In contrast, the FMM background $I_{F M M}^{B S}$ is significantly lower than the FMM signal $I_{F M M}^{B B}$. The SBR ratios are plotted as functions of penetration depth in Fig. 9 (b). It is evident that FMM provides a much more superior SBR than CM even for small imaging depths. For a large imaging depth up to $1 \mathrm{~mm}, S B R_{F M M}$ is maintained at around $20 \mathrm{~dB}$. 

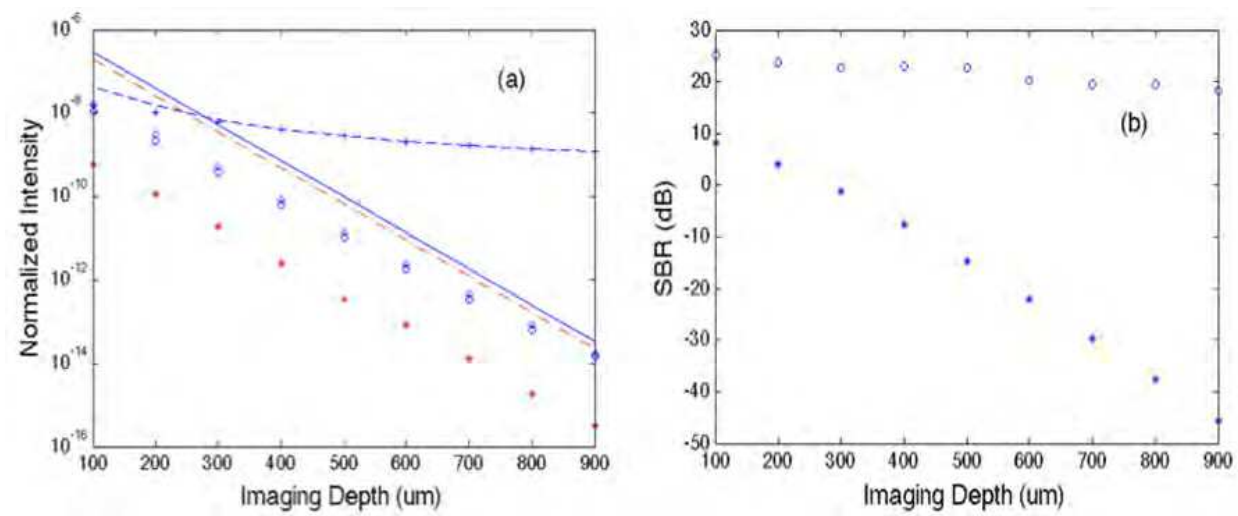

Fig. 9. Normalized intensity and signal-to-background ratio for various depths. (a) FMM signals $I_{F M M}^{B B}$ (dashed-dotted) and $I_{F M M}^{S B}$ (asterisks) compared with CM signals $I_{C M}^{B B}$ (solid), $I_{C M}^{B K}$ (dashed), $I_{C M}^{S B}$ (diamonds), $I_{C M}^{B S}$ (circles), and $I_{C M}^{S S}$ (pluses); (b) SBR for FMM (circles) and CM (asterisks).

In this section, the advantage of FMM is well explained with the theoretical model combining the scalar diffraction theory and Monte Carlo methods. The SBR ratio of FMM is evaluated with such a model and is compared with that of CM. It is obvious that SBR is not a limiting factor for an imaging depth as large as $1 \mathrm{~mm}$. Our model can also be used to analyse other performances such as spatial resolution and SBR ratio of FMM.

\subsection{Effect of aperture configurations on the modulation depth of FMM}

Another important parameter in FMM is the modulation depth, which can be defined as the ratio of the intensity of the modulated fluorescence signal (i.e. the ac component) to the average intensity (approximately the dc component) collected by the aperture pinhole. The modulation depth is crucial in designing illumination aperture for FMM because it determines the efficiency of FMM signal generation with respect to the total excitation power deposited on the sample. Principally, a larger modulation depth will give us enhanced FMM signal and therefore improved signal to noise ratio. In addition, a larger modulation depth allows us to reduce the threshold of excitation power for optical image quality when probing deep into thick living specimens, which is crucial in minimizing the probability of photobleaching and phototoxicity resulted from higher excitation power. In this section, we first define the modulation depth and an analytical expression for its calculation in FMM. In particular, various aperture designs are being considered to compare their effectiveness in generating high modulation depth. The spatial resolutions correspond to each aperture are also presented with some concluding remarks at the end.

\subsubsection{Definition of modulation depth for FMM}

The modulation depth of FMM can be defined as the ratio of the intensity of the modulated fluorescence signal (i.e. ac component) to the average intensity (i.e. dc component) collected by the aperture pinhole when exciting a fluorescence-stained sample:

$$
M=\frac{\frac{1}{2}\left(I_{\max }-I_{\min }\right)}{\frac{1}{2}\left(I_{\max }+I_{\min }\right)}=\frac{I_{\max }-I_{\min }}{I_{\max }+I_{\min }}=\frac{I_{F M M}}{I_{C M}}
$$


Here $I$ denotes the signal intensity at the pinhole detector. In FMM, the illumination apertures consists of, non-modulated and modulated zones, as shown in Fig. 10, in which the green regions having a constant phase delay while the light passing through the blue regions is subject to a time-varying phase delay. The relative phase delay $\varphi(t)$ between the green and blue regions varies from 0 to $\pi$ phase shift. All the apertures are designed such that the total area of the modulated regions equal to that of the unmodulated regions, in order to have the maximum modulation efficiency.

In previous section, we already described the 3D illumination point spread function (IPSF) after the objectives based on scalar diffraction theory. Assuming the wavefront of the illumination beams is uniform before spatial phase modulator, we can obtain the IPSF of $\mathrm{CM}$ and FMM using the following equation in Cartesian coordinates:

$$
E(x, y, z, t)=\left|\iint_{A} P\left(x^{\prime}, y^{\prime}, t\right) \mathrm{e}^{\left\{\frac{i k}{f}\left(x^{2}+y^{2}\right)-\frac{i k}{2 z}\left[\left(x-x^{\prime}\right)^{2}+\left(y-y^{\prime}\right)^{2}\right]\right\}} d x^{\prime} d y^{\prime}\right|^{2}
$$

where $A$ is the open area of the aperture and $P\left(x^{\prime}, y^{\prime}, t\right)$ is the instantaneous phase profile of the spatial phase modulator, which varies with time $t$ according to designated modulation frequency. The lateral and axial coordinates $x, y$, and $z$ are in the image space while $x^{\prime}$ and $y^{\prime}$ indicate the position in the aperture $A$. The wavenumber $k$ is related to the wavelength of the illumination $\lambda$ as $k=\frac{2 \pi}{\lambda}$ and $f$ is the focal length of the objective. Then, the intensity on the detector $I(t)$ can be expressed as:

$$
I(t)=\iiint E(x, y, z, t) \times\left[\left|h_{D}(x, y, z)\right|^{2} \otimes_{2} D(x, y)\right] d x d y d z
$$

where $h_{D}(x, y, z)$ is the amplitude point spread function of the detection optics, $D(x, y)$ is the sensitivity function for the detector and is related to the size of the aperture pinhole in front of the detector. Thus, when the phase over the apertures is uniform (i.e. $\varphi(t)=0$ ), a strong constructive interference at the focal spot of the objectives is expected and the detected signal at the central focal spot will reach the maximum, which is also identical to that of CM. For $\mathrm{CM}$, the phase profile is uniform (i.e. $\left.P\left(x^{\prime}, y^{\prime}, t\right)=1\right)$ and thus the IPSF is simply:

$$
E_{C M}(x, y, z)=\left.\left|\iint_{A} P\left(x^{\prime}, y^{\prime}, t\right)\right|_{\varphi(t)=0} \mathrm{e}^{\left\{\frac{i k}{f}\left(x^{2}+y^{2}\right)-\frac{i k}{2 z}\left[\left(x-x^{\prime}\right)^{2}+\left(y-y^{\prime}\right)^{2}\right]\right\}} d x^{\prime} d y^{\prime}\right|^{2}
$$

Then, the detected signal of $\mathrm{CM}$ at the focal spot is:

$$
I_{C M}=\iiint E_{C M}(x, y, z) \times\left[\left|h_{D}(x, y, z)\right|^{2} \otimes_{2} D(x, y)\right] d x d y d z
$$

On the other hand, when the relative phase delay of the modulated and unmodulated beams $\varphi(t)$ is equal to $\pi$, a strong destructive interference will occur at the central spot of the point spread function of the illumination beams (i.e. within the first zeroth of the IPSF of FMM) and the detected intensity approximately reach the minimum (i.e. $I_{\text {min }}$ ), with highintensity side lobes appearing immediately outside the central focal spot. At such, we can define the effective FMM intensity point spread function as the difference of the intensity at the central focal spot for illumination with uniform phase (i.e. $I_{\max }$ ) and that of modulated phase apertures (i.e. $I_{\min }$ ); which can be easily shown to be proportionally equal to the FMM intensity detected using heterodyne detection in real implementation. Here, we outline the equations for the illumination PSF of FMM: 


$$
E_{F M M}(x, y, z)=E_{C M}(x, y, z)-\left.\left|\iint_{A} P\left(x^{\prime}, y^{\prime}, t\right)\right|_{\varphi(t)=\pi} \mathrm{e}^{\left\{\frac{i k}{f}\left(x^{2}+y^{2}\right)-\frac{i k}{2 z}\left[\left(x-x^{\prime}\right)^{2}+\left(y-y^{\prime}\right)^{2}\right]\right\}} d x^{\prime} d y^{\prime}\right|^{2}
$$

Then, the FMM intensity is simply:

$$
I_{F M M}=\iiint E_{F M M}(x, y, z) \times\left[\left|h_{D}(x, y, z)\right|^{2} \otimes_{2} D(x, y)\right] d x d y d z
$$

Finally, the modulation depth of FMM can be easily calculated using equations. It is important to understand that the total intensity loading on the focal plane inside the sample (as well as the whole sample) is always uniform regardless of the relative phase-delay of the patterned illumination beam, and the detection pinhole (as well as its size) is crucial in collecting only the intensity-modulated fluorescence emission from the focal volume. Else, the amplitude of FMM signal over the focal plane would drop significantly.

\subsubsection{Simulation results}

The simulation results based on analytical expressions described in the previous section are presented here. Typical system parameters are being used in our simulation. The laser wavelength is $633 \mathrm{~nm}$. For simplicity, we assume the objective lens $L_{1}$ and the collection lens $\mathrm{L}_{2}$ are identical and have the same numerical aperture of 0.55 , and the excitation and emission lights have same wavelength as well. The pinhole diameter used is $0.8 \mu \mathrm{m}$, about $0.6 d_{\text {Airy }}\left(d_{\text {Airy }}\right.$ is the diameter of Airy disk). Eight apertures (see Fig. 10), which includes three annular apertures, three fan-shaped apertures and two stripe-shaped apertures, are investigated. These apertures are selected partly due to their ease of fabrication.

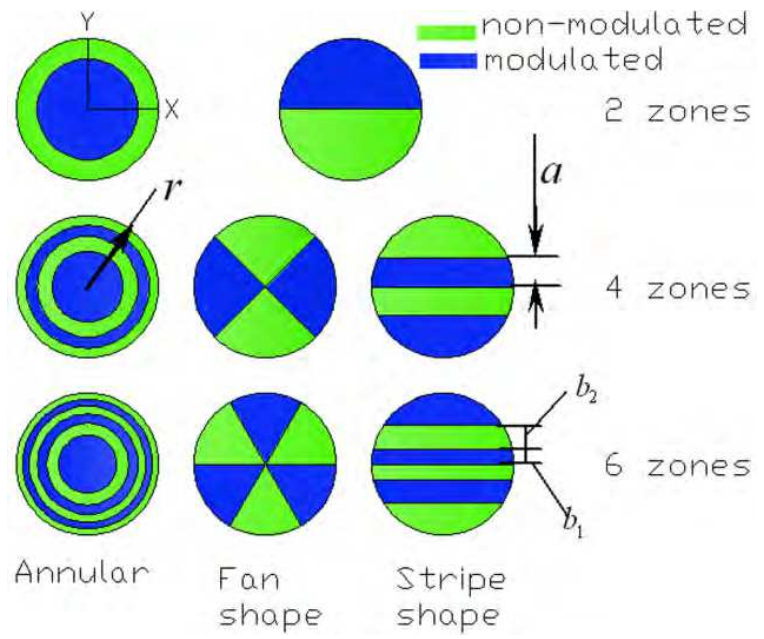

Fig. 10. Configurations of SPM apertures that are being investigated. The areas with green color are non-modulated, and those with blue are modulated. According to the first principle of aperture selection, the internal radii of annular apertures are $\frac{r}{\sqrt{2}}$ for 2-zone annular aperture, and $\frac{r}{2}, \frac{r}{\sqrt{2}}, \frac{\sqrt{3} r}{2}$ for 4-zone annular aperture, $\frac{r}{\sqrt{6}}, \frac{r}{\sqrt{3}}, \frac{r}{\sqrt{2}}, \frac{\sqrt{6} r}{3}, \frac{\sqrt{30} r}{6}$ for 6-zone annular aperture. Similarly, the widths for stripe apertures are $a \approx 0.404 r$ for 4-zone stripe aperture and $b_{1} \approx 0.215 r, b_{2} \approx 0.338 r$ for 6-zone stripe aperture. $r$ is the external radius of aperture. 
The effective illumination IPSFs for different apertures calculated using equations described in section 4.4.1 are presented in Fig. 11. They are displayed as enface (upper row) and crosssectional (lower row) 2D images of around the focal point. Fig. 11 (a) are the effective IPSFs for annular apertures of two zones (left column), four zones (middle column), and six zones (right column), respectively. The results for fan-shaped and stripe-shaped apertures are arranged in a similar way in Fig 11 (b) and (c). The coordinates of the Figs $v_{x}, v_{y}$ and $u$ are related to the practical coordinates $x, y$, and $z$ by $v_{x}=k N A x, v_{y}=k N A y$ and $u=4 k\left[\sin \frac{\alpha}{2}\right]^{2} z$ with $N A$ the numerical aperture of objective lens, $\alpha$ the semi-angle aperture of the objective lens. The illumination IPSFs of FMM can be negative for some regions outside central focal volume due to differential excitation mechanism of FMM. If these negative side lobes are allowed to be included in the FMM image formation (i.e. by enlarging the detection pinhole), the FMM signals will diminish and become ineffective (Chen, Wong et al. 2008).
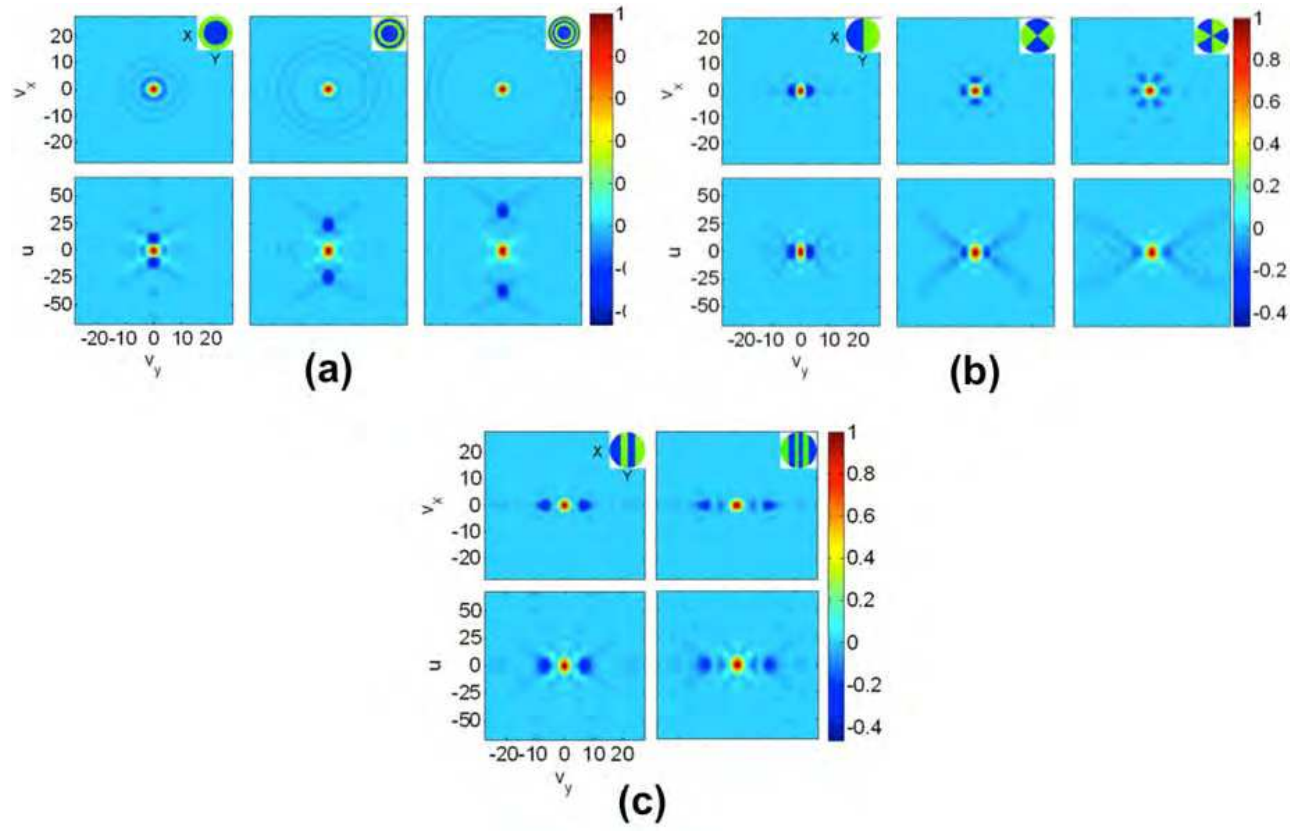

Fig. 11. IPSFs for (a) annular, (b) fan shape and (c) stripe shape apertures. In (a) and (b) the first, second and third columns are corresponding to two, four, and six zones. In (c) The result of two zones is omitted because it is the same as the two-zone fan-shape aperture. The icon on the top-right of each graph is the corresponding aperture shape.

From the IPSFs in Fig 11, we can calculate the modulation depth using the method described in previous section. The calculated modulation depth for the investigated apertures is shown in Table 2. Additionally, the lateral and axial spatial resolutions achievable are also expressed in term of full width half maximum (FWHM) of the simulated IPSFs (see Table 3), which is the diameter of the central spot where the intensity drops beyond half of the maximum. Basically, there are no significant differences between the achievable spatial resolutions in both lateral and axial directions. 


\begin{tabular}{|c|c|c|c|}
\hline \multirow{2}{*}{ Aperture pattern } & \multicolumn{3}{|c|}{ Number of zones } \\
\cline { 2 - 4 } & $\mathbf{2}$ & $\mathbf{4}$ & $\mathbf{6}$ \\
\hline Annular & 0.446 & 0.757 & 0.865 \\
\hline Fan & 0.361 & 0.602 & 0.762 \\
\hline Stripes & 0.361 & 0.691 & 0.814 \\
\hline
\end{tabular}

Table 2. Modulation depths for various aperture patterns.

\begin{tabular}{|c|c|c|c|c|c|c|}
\hline & \multicolumn{5}{|c|}{ Lateral } & \multicolumn{4}{c|}{ Axial } \\
\hline \multirow{2}{*}{ Aperture pattern } & \multicolumn{5}{|c|}{ Number of zones } \\
\cline { 2 - 7 } & $\mathbf{2}$ & $\mathbf{4}$ & $\mathbf{6}$ & $\mathbf{2}$ & $\mathbf{4}$ & $\mathbf{6}$ \\
\hline Annular & 2.666 & 2.689 & 2.692 & 6.692 & 7.699 & 7.898 \\
\hline Fan & 2.694 & 2.697 & 2.697 & 8.057 & 8.056 & 8.057 \\
\hline Stripes & 2.694 & 2.696 & 2.696 & 8.057 & 8.060 & 8.059 \\
\hline
\end{tabular}

Table 3. Lateral and axial spatial resolution for various aperture patterns.

\subsubsection{Discussions}

We have investigated the effectiveness of various aperture patterns in achieving higher modulation depth for FMM that are important for efficient generation of intensity modulation at the focal spot. We compare the resultant modulation depths and achievable spatial resolutions for these aperture patterns through analytical calculations, with several comments can be made.

First, with the number of zones increasing from 2 to 6 , the modulation depths of the three types of apertures increase from $0.3 \sim 0.4$ to $0.75 \sim 0.85$, as shown in Table 2 . As explained earlier, there are negative side lobes in the effective IPSFs as FMM is based on differential excitation mechanism (see Fig. 11). In the cases for an aperture with two zones, the negative side lobes appear very close to the positive central main lobe and their absolute peak values are greater than 0.4 (normalized to the main lobe peak value). Such behaviour lead to reduced FMM signal if these negative side lobes are included in FMM image formation when the pinhole size is too large. Alternatively, we can implement smaller detection pinhole to exclude these negative side lobes, which at the same time reduced FMM signals from central spot. By increasing the number of zones in the SPM aperture, however, we can disperse the negative side lobes, moving their peak positions slightly away from the focus and at the same time reducing their magnitudes. Thus, we have greater flexibility in selecting larger detection pinhole to capture maximum FMM signals from central focal spot. This is generally true for annular, fan-shape, and stripe-shape apertures.

Second, it is observed that there is slight degradation (from 6.7 to 7.9 ) for the axial resolution of annular aperture when the modulation depth is being increased by increasing the number of zones from 2 to 6, and almost no influence to the axial resolutions of other type of apertures, which is shown in Table 3. Previously, it has been shown that CM with an annular aperture and a point detector will result in improvement of lateral spatial resolution at the cost of degradation of optical sectioning strength (i.e. axial resolution) (Sheppard and $\mathrm{Gu}$ 1991). However, using a finite sized pinhole, annular aperture could give us improvement of axial resolution (Gu and Sheppard 1991). The effect of size of pinhole 
detector on the axial resolution of FMM with annular aperture as the number of zones increase is thus worth to be further investigated in future.

Last but not least, from the standpoint of modulation depth and axial resolution, the annular apertures are superior to the fan shape and stripe shape apertures, while the achievable lateral resolutions are approximately the same for all types of apertures. This is expected because neither the fan-shape nor the stripe-shape apertures have noticeable negative side lobes in the axial direction (Fig. 11 (b) and (c)), except the annular aperture that results in strong negative side lobes along the optical axis (Fig. 11 (a)), but has been taken care off by the pinhole. There are strong side lobes in the focal plane for the fan-shaped and stripeshaped apertures due to their circular asymmetry and therefore better resolving power is expected in certain directions, such as, along $y$ direction for fan shape and stripe shape apertures (see Fig. 11 (b) and (c)). Nevertheless, the poorest resolution along the lateral directions are being chosen as comparison. At such, we found no significant improvement of the lateral resolution for all apertures.

\section{Implementations of focal modulation microscopy}

Here, we describes on the implementations of two spatiotemporal phase modulation schemes for focal modulation microscope - double reflecting mirrors as well as FMM based on acousto-optical modulators that are significantly progressive in term of stability and image performance.

\subsection{Double-reflecting mirror}

\subsubsection{System design}

Shown in Fig. 12 is the schematic diagram of the first prototype FMM system developed at the Optical Bioimaging Lab, National University of Singapore. The light source is a $640 \mathrm{~nm}$

solid state single frequency laser, whose $25 \mathrm{~mW}$ output beam is expanded to about $5 \mathrm{~mm}$ in diameter. When passing through the spatial phase modulator, the beam is split into two spatially separated half-beams, which are parallel and subject to different phase delays. The spatiotemporal phase modulation is implemented with two parallel mirrors (M1 and M2) inside the dashed box. M1 is mounted on a stationary base while M2 is mounted on a piezoelectric actuator. A sinusoidal voltage signal of a single frequency $f=5 \mathrm{kHz}$ superimposed on an appropriate dc bias is applied to the actuator to induce a relative phase shift periodically between 0 and $\pi$. The spatiotemporal phase modulated excitation beam is reflected by a dichroic mirror and is then directed by a two-dimensional fast steering mirror to a 20X objective. Fluorescence emission is collected by the same objective and de-scanned is performed by the same fast steering mirror. A long pass emission filter is used to further reject the excitation light already suppressed by the DM. The cut-on wavelength for both the $\mathrm{DM}$ and the long pass filter is between 667 and $670 \mathrm{~nm}$. Then the fluorescence light is focused by an achromat and coupled into a single mode (SM) optic fiber. A photomultiplier tube (R928, Hamamatsu Photonics Co.) converts the weak light signal to an electrical signal, which is digitized and sampled by a DAQ card in a personal computer (PC). The acquired photoelectrical signal contains a constant DC component and a time-varying AC component at $5 \mathrm{kHz}$ due to modulated excitation. A Fast Fourier Transform (FFT) is performed on the PC to retrieve both FMM and CM signals respectively. 


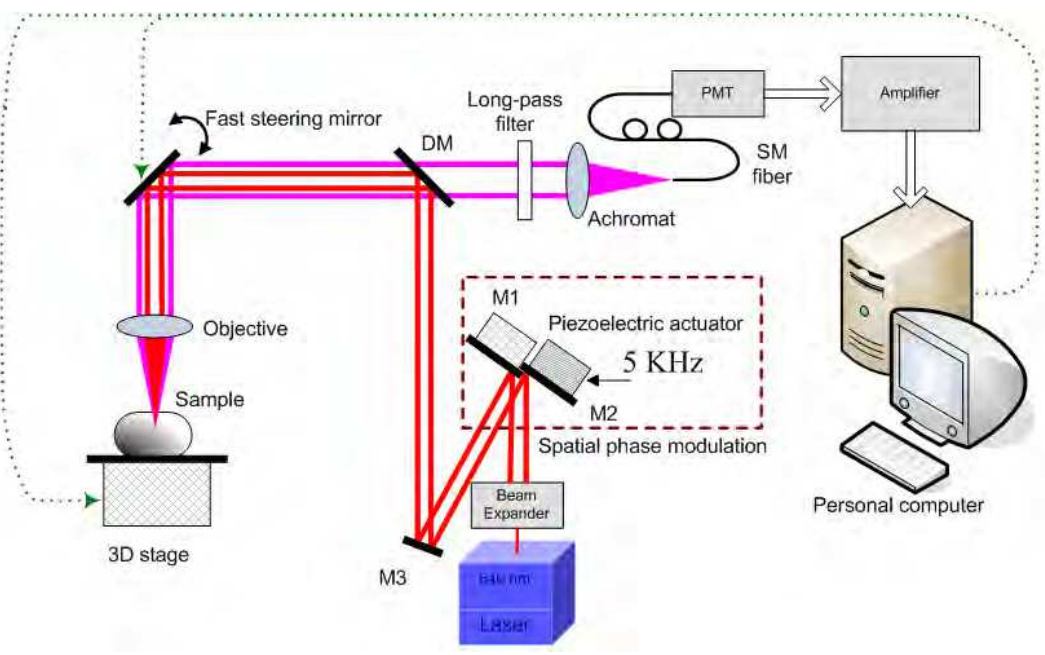

Fig. 12. Schematic diagram of the prototype focal modulation microscopy system. The spatial phase distribution of the $640 \mathrm{~nm}$ excitation beam (red) is modulated by the use of two parallel mirrors, M1 (stationary) and M2 (oscillating axially at $5 \mathrm{kHz}$ ). The fluorescence emission (purple) from the focal volume is collected by a fiber based confocal detection system, and then the oscillatory component at $\mathbf{5} \mathrm{kHz}$ is retrieved for image formation. The personal computer is used for data acquisition and analysis, lateral scanning with the fast steering mirror, and axial scanning with the 3D stage.

\subsubsection{Experiments and results}

To demonstrate the capability of our method for in vivo imaging of cellular and sub-cellular structure and function, we used chicken cartilage as a sample tissue to evaluate the performance of FMM. Chondrocytes are the only cells found in cartilage. The cells are usually of a rounded or bluntly angular form, lying in groups of two or more in a glandular or almost homogeneous matrix. Chicken cartilage was cut into slices around $\mathbf{1} \mathrm{mm}$ in thickness and stained with $\mathrm{DiD}$ (DiIC18 (5), Invitrogen Corp.), a lipophilic tracer for cell membrane labeling. The laser power was attenuated by $10-1000$ times to avoid fast photobleaching. Fig. 13 (a) and 13 (b) are CM and FMM images acquired at a depth around $280 \mu \mathrm{m}$.

One can easily see that optical sectioning is not effective in the confocal image (Fig 13(a)). Fluorescence signals from layers other than the focal plane cast shadows in the image, resulting in overlapping structures and blurred cellular shapes. On the contrary, the FMM image (Fig. 13 (b)) shows uncompromised quality. The upper-central area was then scanned with a four times finer step (about $100 \mathrm{~nm}$ ) and the high magnification images are displayed in Fig. 13 (c) and (d). The FMM image (Fig. 13 (d)) provides detailed information with submicron spatial resolution and excellent contrast, which are not available from the CM image (Fig. 13 (c)). We then pushed further to test the maximal penetration depth of our prototype FMM system. Shown in Fig 13. (e) and (f) are FMM images from 500 and $600 \mu m$ in depth. It is evident that resolution is still high enough to visualize cellular structures. Beyond $600 \mu \mathrm{m}$, the shot noise associated with the background will gradually overwhelm the FMM signal. 
To the best of our knowledge, the penetration depth we report here has never been achieved by one photon fluorescence based microscopy when imaging optically dense biological tissues. However, there might be still room for further improvements. The tissue samples in our experiments were uniformly stained. As a result, the superficial layers strongly absorbed the excitation light and generated intense background emissions. For certain applications where only the deep region of interest is stained, an even larger penetration depth should be possible. More photostable and brighter fluorescent labels (e.g., quantum dots) can also help further increase the imaging depth.
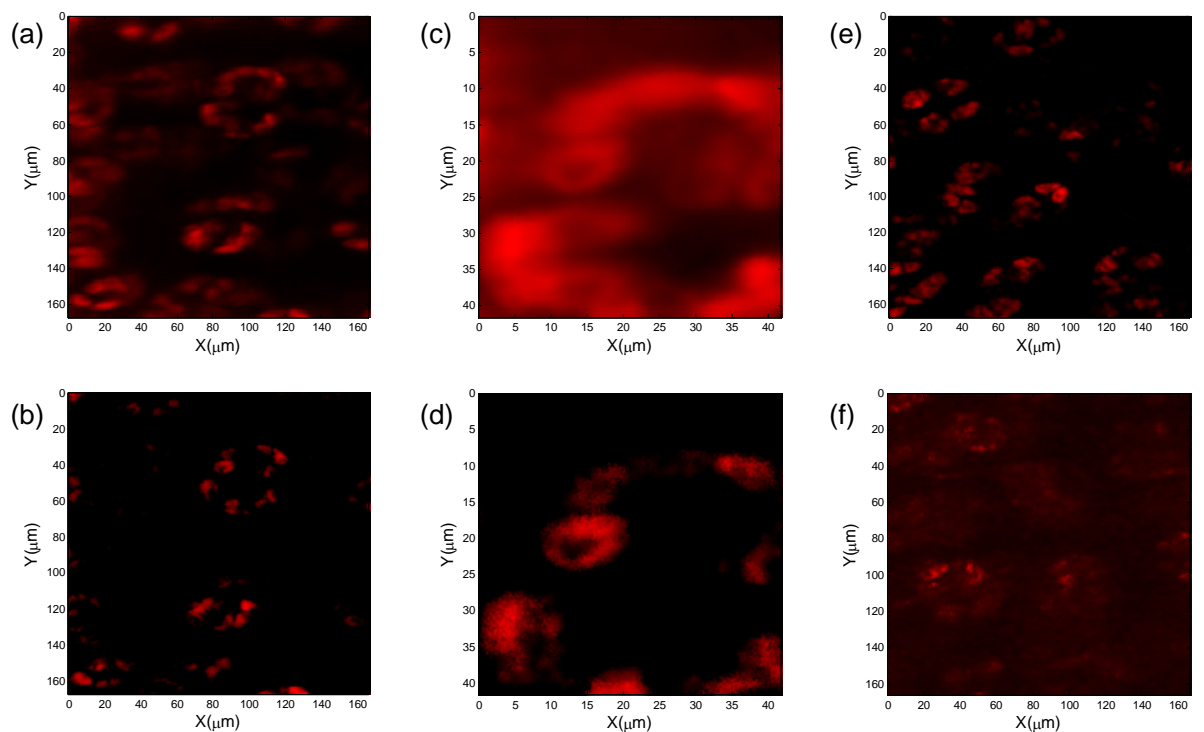

Fig. 13. Fluorescence images of chondrocytes obtained from chicken cartilage. Confocal images $((\mathrm{a})$ and $(\mathrm{c}))$ were acquired simultaneously with the corresponding FMM images ((b) and (d)) at a depth of $280 \mu m$. (e) and (f) are FMM images obtained from 500 and $600 \mu m$ in depth.

The imaging depth mentioned above was achieved with a relatively long excitation wavelength of $640 \mathrm{~nm}$. For excitation light of shorter wavelength, such as UV or blue light, the achievable imaging depth is expected to be smaller due to stronger scattering. On the other hand, near infrared fluorescence dyes (Indocyanine Green, for example) would allow the use of an even longer excitation wavelength $(780 \mathrm{~nm})$ and deeper imaging. The imaging speed of this prototype FMM is limited by the spatiotemporal phase modulator consisting of two mirrors. The minimal dwelling time at each pixel is $0.2 \mathrm{~ms}$, significantly longer than the typical 10 microsecond pixel dwelling time for commercial CM and MPM systems.

\subsubsection{Discussions}

The imaging depth demonstrated here is related to a relatively long excitation wavelength of $640 \mathrm{~nm}$. Generally the scattering coefficient of biological tissue decreases with increasing wavelength, and in many situations the attenuation of ballistic light is dominated by scattering. For excitation light of shorter wavelength, such as UV or blue light, the 
achievable imaging depth should be smaller. On the other hand, near infrared fluorescence dyes (Indocyanine Green, for example) would allow the use of an even longer excitation wavelength $(780 \mathrm{~nm})$ and deeper imaging. In our future research, we will systematically study the dependence of imaging depth on excitation wavelength and fluorescence dye properties. The imaging speed of our prototype FMM is limited by the modulation frequency. The minimal dwelling time at each pixel is $0.2 \mathrm{~ms}$, significantly longer than the typical $10 \mu$ s pixel dwelling time for commercial CM and MPM systems. We plan to develop electro-optical material based spatial phase modulators that can be modulated at a frequency more than a few MHz. Near-real-time FMM imaging (a few frames per second) would be possible with such a modulator.

\subsection{FMM using acousto-optical modulators}

We reported our FMM system implemented using tilting plate phase modulator (Wong, Chong et al. 2009) where a modulation frequency up to a few tens of $\mathrm{kHz}$ can been achieved. However, compared to most commercial laser scanning microscopes with microsecond pixel dwell time, that modulation frequency is still too low. In this section we describe a near-realtime FMM with AOMs and demonstrate the improved image acquisition speed and quality. Our FMM system is based on an Olympus FV300 confocal microscope

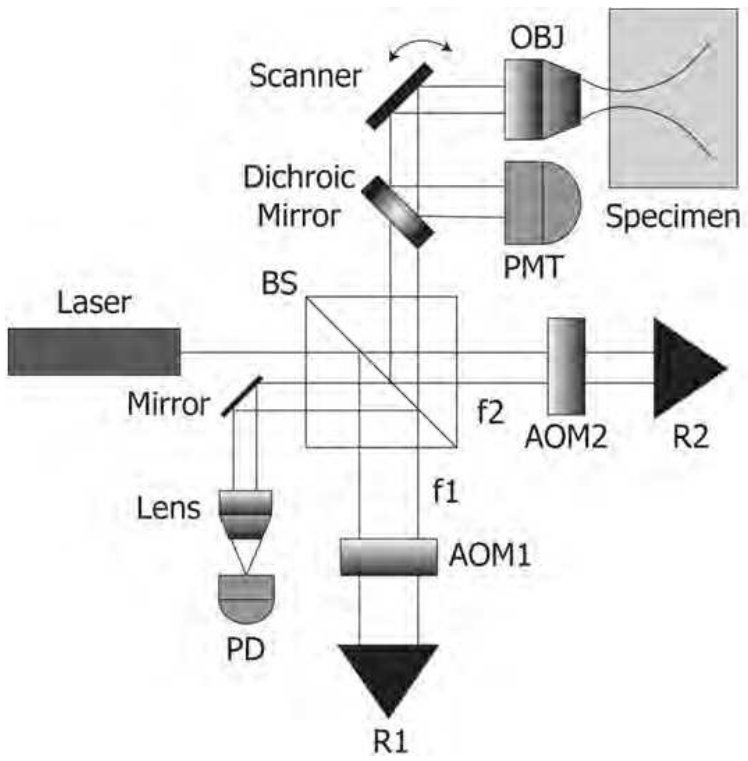

Fig. 14. Setup for a FMM implemented using acousto-optical modulators. Laser beam is split by a beam splitter (BS) in which light beam in each arm undergoing frequency shifting by two acoustic-optical modulators (AOM) with different resonance frequencies. Phase-shifted beams are reflected back to the BS by retroreflectors (R1 and R2). They are aligned in parallel non-overlapping manner when being split second times at BS in which one combined beam will be used to generate a reference signal while another combined beam is directed to the scanning unit of Olympus FV300 to excite the samples through the objective lens (OBJ). PD is a photodetector and $\mathrm{M}$ is a mirror. 


\subsubsection{Optical setup}

The spatio-temporal phase modulator based on AOMs is shown schematically in see Fig. 14 . The coherent laser beam is first split by a beam splitter into two beams which then passes through two AOMs with slightly different resonance frequencies (i.e. $f_{1}$ and $f_{2}$ ) where they undergo zeroth- and first-order diffractions. The first-order diffracted beams which are Doppler shifted at the $f_{1}$ and $f_{2}$ on each arm. They are reflected with slight lateral displacement using retroreflectors to return to the same AOMs and then are recombined at beam splitter. It is important to note that the two beams at the moment are Doppler shifted at twice the resonance frequencies of the AOMs respectively as they passed through the AOMs twice. Part of the combined beam is directed towards a fiber-optic photodetector, which generates a reference signal at the optical beating frequency of $2\left(f_{1}-f_{2}\right)$. The remaining part of the modulated laser beam is directed to the scanning unit of a conventional $\mathrm{CM}$ (Olympus FV300) to excite the biological sample stained with fluorescence probes.

\subsubsection{Detection of FMM signal}

The fluorescence emissions are then detected by the built-in PMT detector (R3896, Hamamatsu Photonics Co.) behind a confocal pinhole. The PMT output is preamplified before feeding to an I/Q demodulator, where the oscillatory component at the beating frequency is picked up by mixing with the reference signal. The demodulated signal is further enhanced by the low-frequency amplifier and is then fed to the data acquisition device of the FV300 system (see Fig. 15 for the signal processing pathway).

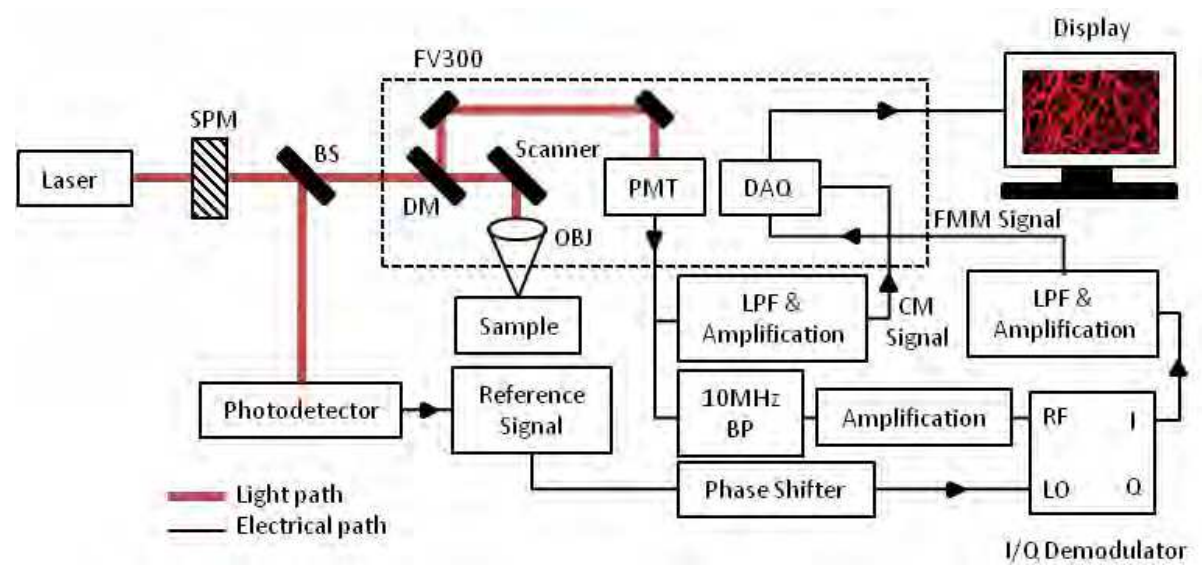

Fig. 15. Flow diagrams showing the signal-processing pathway.

The two AOMs we are using have resonant frequencies of $75 \mathrm{MHz}$ and $80 \mathrm{MHz}$, respectively, resulting in a modulation frequency of $10 \mathrm{MHz}$. In principle, such a high modulation frequency allows a pixel dwell time as short as 0.1 microseconds. This instrument is a significant improvement in term of imaging speed, stability and noise level compared with the prototype setup we reported previously (Chen, Wong et al. 2008; Wong, Chong et al. 2009). CM and FMM images can be captured simultaneously with the same acquisition speed using our current system. The effectiveness of FMM in improving image quality is demonstrated by imaging blood vessels in mouse brain labeled with lipophilic 
carbocyanine dye DiI ('DiI'; DiIC18(3), Invitrogen), self-made tissue phantom as well as DiD stained chicken chondrocytes.

\subsubsection{Experiments and results}

The mouse brain samples were prepared using the established techniques described in the literature (Li, Song et al. 2008). A mouse was first sacrificed by overdose with $\mathrm{CO}_{2}$ in a $\mathrm{CO}_{2}$ chamber. A transverse incision was made to open the abdominal cavity. The diaphragm was exposed and cut. The chest was then cut on both sides up to the second rib. The anterior chest wall was turned toward the head of the mouse to expose the chest cavity. The butterfly needle of the perfusion device was inserted into the left ventricle, and the right atrium was punctured. $2 \mathrm{ml}$ of standard phosphate buffer solution (PBS) was injected from the perfusion device at the rate of $1-2 \mathrm{ml} / \mathrm{min}$ for $5 \mathrm{~min}$. Then, $5-10 \mathrm{ml}$ of the DiI solution was injected at a rate of $1-2 \mathrm{ml} / \mathrm{min}$ for $5-10 \mathrm{~min}$. Successful perfusion was monitored by observing color change in the ear, nose and palms of the mouse from pale while to purple. Lastly, the fixative (4\% paraformaldehyde solution) was injected at a rate of $1-2 \mathrm{ml} / \mathrm{min}$ for a total of $5 \mathrm{~min}$. It should be ensured that there are no air bubbles in the perfusion device as introduction of air into the circulatory system could block blood vessels, resulting in poor perfusion. The stained mouse brain was then extracted, and stored in $4 \%(\mathrm{wt} / \mathrm{vol})$ paraformaldehyde solution. The whole fixed mouse brain is mounted by mounting medium (Polyvinyl alcohol mounting medium with $\mathrm{DABCO}^{\circledR}$, antifading BioChemika, SigmaAldrich) in a coverslip bottom dish with cover (MatTek Corp) for imaging using our FMM.

Besides fluorescence imaging, we also apply our FMM system on imaging a cylindrical selfmade tissue phantom slab mainly consisted of epoxy-resin, resin hardener, and titanium dioxide $\left(\mathrm{TiO}_{2}\right)$. The $\mathrm{TiO}_{2}$ powder acts as scattering components and the main contrast agent inside the tissue phantom. This tissue phantom was made based on the procedures reported in literature (Firbank, Oda et al. 1995) and is commonly used for calibration in diffuse optical tomography (DOT) (Mo and Chen 2008). The chicken cartilage sample is prepared in the similar way described in section 5.1.2.

\section{A. Mouse brain vasculatures imaging}

The mouse brain samples were imaged using our FMM system with either objectives UPLSAPO 10x/0.40 numerical aperture (NA) or LUCPLANFLN 20x/0.45 NA to evaluate our FMM system in term of image quality improvement. Here we show the images captured using CM and FMM simultaneously to demonstrate the robustness of FMM in rejecting outof-focus fluorescence background and retaining high-resolution features even imaging deep inside tissues (depicted by Fig. 16).

A blood vessel bifurcation imaged using CM (Fig. 16 (a)) and FMM (Fig. 16. (b)) shows that much finer features can be visualized by FMM clearly at the penetration depth around 200um. Fig. 16 (c) and (d) shows the digital magnification near the bifurcation of the main blood vessel (indicated by white boxes in Fig. 16 (a) and (b)) which also reveals better contrast on the fine structures of the blood vessels by the image captured by FMM as compared to CM. From the intensity profiles spanning the both white lines (top-down) in the Fig. 16 (c) and (d), we can also observe that the peaks are more clearly visible and distinctive from each other's in the FMM image with sharper valleys, and significant reduction in background as well. This indicates that our FMM system can provide better image contrast and more structural information of the tissue being studied. 

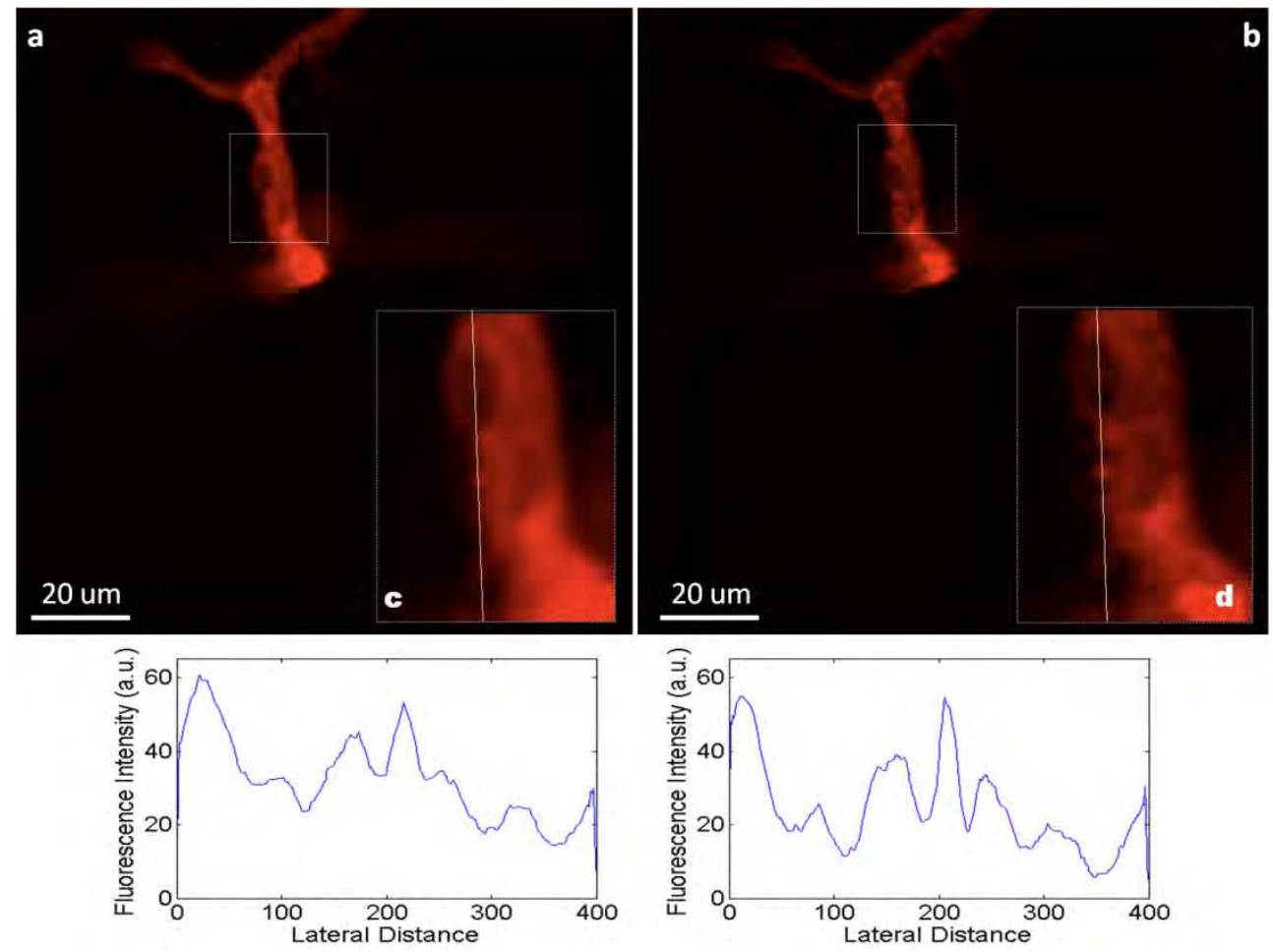

Fig. 16. Demonstration of FMM in retaining high-resolution features by rejecting out-offocus fluorescence background. A blood vessel bifurcation image acquired with Olympus LUCPLANFLN 20x/0.45 NA using (a) CM and (b) FMM shows much finer features can be revealed by FMM even at penetration depth up 200um. Fig. 16 (c) and (d) shows the digital magnification near the bifurcation of the main blood vessel which reveals much richer features on the image captured by FMM. The plot at the bottom of the images shows the intensity profiles of the white lines (top-down) in the images.

\section{B. Tissue phantom imaging}

We also performed imaging in the tissue phantom over the depths of $300-400 \mu \mathrm{m}$ with $0.5 \mu \mathrm{m}$ increments. A single frame image of a cluster of $\mathrm{TiO}_{2}$ powder (see Fig. 17) embedded at $320 \mu \mathrm{m}$ inside the self-made tissue phantom captured using CM and FMM operated in reflectance mode.

Similarly, FMM (see Fig. 17 (b)) could significantly improve the image quality by providing superior contrast and sharp edges as compared to $\mathrm{CM}$ which are blurred mainly by multiple scattering effects. The peaks are also more clearly distinguished from each other in FMM despite the $\mathrm{TiO}_{2}$ granules are in close proximity, as shown by the intensity profile spanning the white line (left-right), with higher SBR ratio due to lower background intensity. 

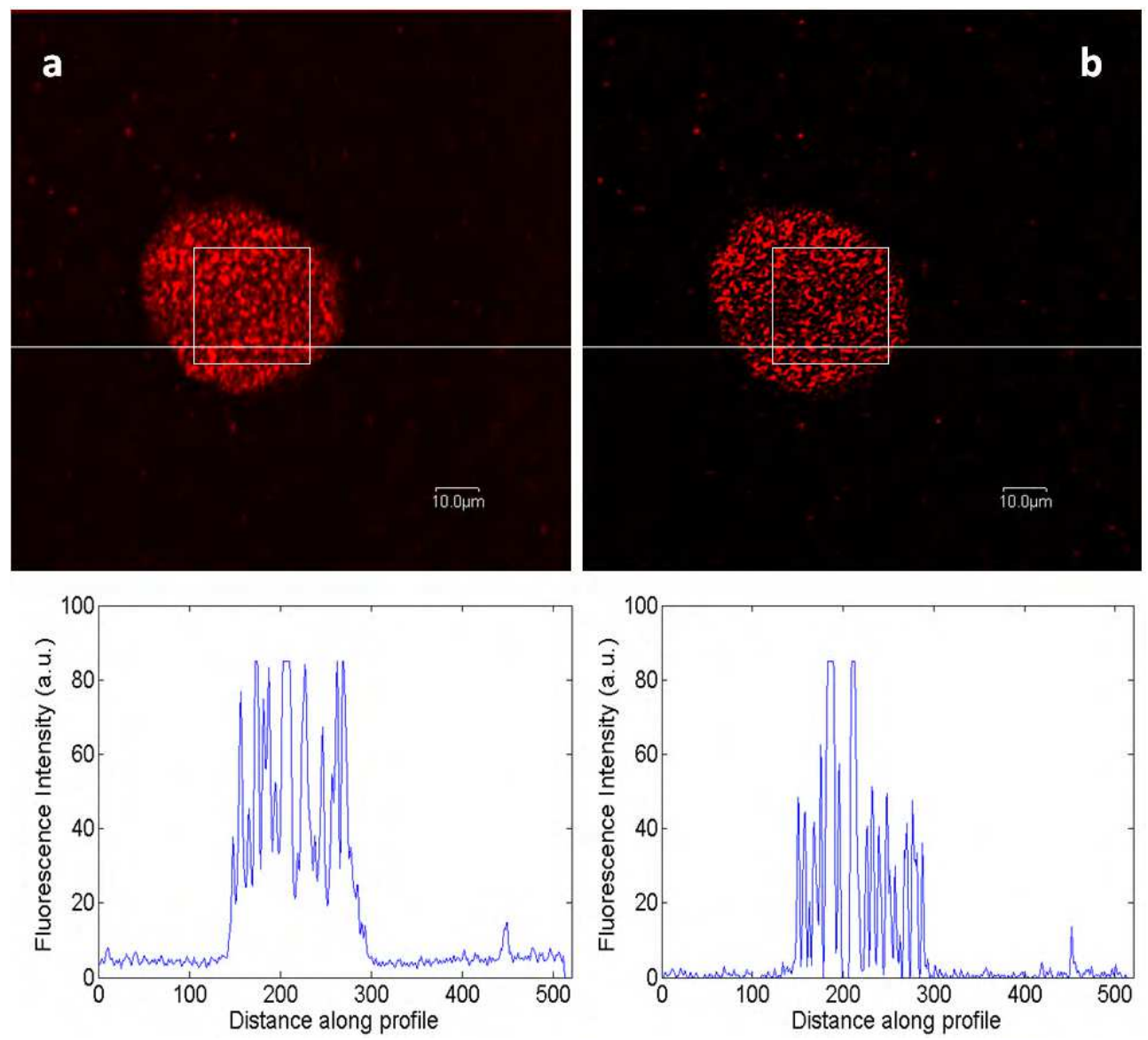

Fig. 17. Image of cluster of $\mathrm{TiO}_{2}$ embedded $320 \mathrm{um}$ inside tissue phantom captured with Olympus LUCPLANFLN 20x/0.45 NA using (a) CM and (b) FMM. Better image quality is achieved using FMM, with less blurring and structures are more clearly distinguishable due to sharp boundaries. The plot at the bottom of the images shows the intensity profile of the white lines in the images. Peaks are more distinct with better contrast can be observed.

To perform more quantitative analysis of image quality improvement by FMM, we selected a pair of identical region of interest (ROI) from Fig. 17 (a) and (b) respectively and compare their normalized spectra (with respect to the dc values). From Fig. 18, we can observe that the high frequency components $(>1$ cycle $/ \mu \mathrm{m})$ in the FMM image are about $13 \mathrm{~dB}$ stronger than those in the CM image. This can be translated to a $13 \mathrm{~dB}$ improvement in the SBR ratio provided by FMM. However, our previous theoretical model (Chong, Wong et al. 2010) predicted a $20 \mathrm{~dB}$ enhancement by FMM at a similar imaging depth of 2 scattering mean free paths (the scattering coefficient of our tissue phantom is around $6 \mathrm{~cm}^{-1}$ (Mo and Chen 2008). This discrepancy could be due to slight overlapping of the two excitation beams and the resultant residual intensity modulation which can be minimized by improved modulator designs. 


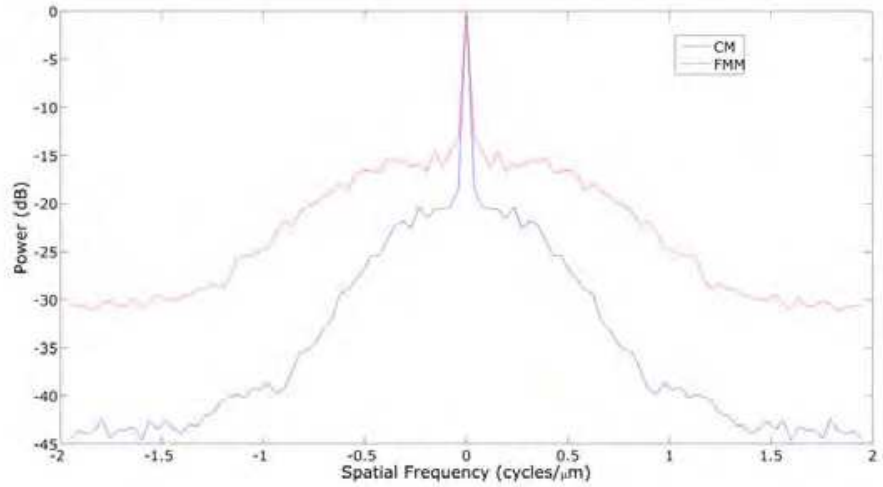

Fig. 18. Normalized spatial spectra (with respect to dc values) of the region of interests labeled by square boxes in Fig. 17 (a) and (b) showing the spatial frequency components of $\mathrm{CM}$ and FMM respectively.

\section{Chicken chondrocytes samples}

We also imaged chondrocytes of the chicken cartilage samples labelled with lipophilic carbocyanine dye DiD (DiD' solid; DiIC18 (5) solid, Invitrogen) using objectives UPLSAPO 10x/0.40 numerical aperture (NA).

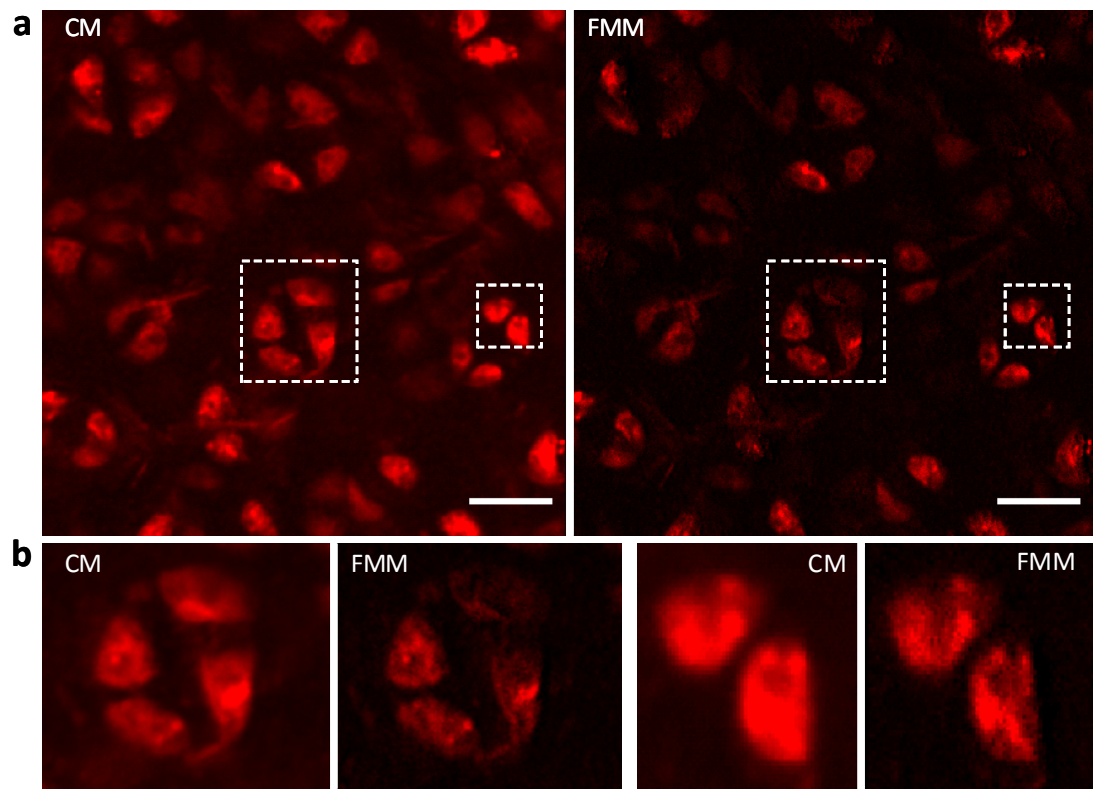

Fig. 19. Comparison of FMM and confocal fluorescence microscopy of chondrocytes of chicken cartilages at penetration depth around $350 \boldsymbol{\mu m}$. (a) The chondrocytes were labeled with DiD (red). (b) Magnifications of the indicated regions. Scale bars $\mathbf{2 0} \boldsymbol{\mu m}$. 
Generally, it is evident that the FMM image gives us less background than raw confocal fluorescence microscopy image (Fig. 19 (a)). The optical contrast of FMM image is also greatly improved, whereas the confocal data are blurred as a result of light scattering. The removal of scattered light also translated directly into enrichment in information quality, revealing features inside the cellular compartments that were obscured in confocal images (Fig. 19 b), indicating that FMM can preserve the optical contrast for large penetration depth in which the fluorescence background dominates the collected signals. The advantages of FMM in terms preserving optical contrast and providing richer subcellular information will be important for imaging of subcellular studies of thick specimens such as characterizing the morphological changes of nuclei in cancers cells (Yao, Maslov et al. 2010).

\subsubsection{Discussions and summary}

We have validated the capability of FMM in improving image quality at large penetration depth by visualizing various specimens. All the images were taken at $512 \times 512$ pixels, with a pixel dwell times of around $10 \mu \mathrm{s}$, resulting image acquisition time of around $2.7 \mathrm{~s}$. We emphasized that as the mouse brain samples are specifically stained in which only blood vessels void of blood (one of the major absorbers and scatterers inside biological tissues) are labeled with $\mathrm{DiD}$, generally less background will be observed while larger penetration depth is expected compared to uniformly stained samples. Nevertheless, CM still suffers from poor image quality especially in observation of finer features; while FMM can work robustly in detecting fluorescence by ballistic photons and rejecting out-of-focus fluorescence background even at the large depth. This is an important feature for investigations and characterizations of thick tissue with sub-micron spatial resolution when confocal pinhole fails to reject majority of the out-of-focus fluorescence background. Better characterization of microvasculatures in animal model over large observation volume using FMM can be potentially useful especially for quantification of angiogenesis in term of density/diameter of blood vessels in live animal cancer model (Lunt, Gray et al. 2010). We also highlight that though FMM can reject most of the background, it cannot increase the signal level and thus if the FMM signal falls below the shot noise floor at extreme depths, other strategies (i.e. denoise image processing and/or extended image acquisition time) may be needed to improve the image quality.

A modulation frequency of $160 \mathrm{MHz}$ can be achieved if only a single AOM is used. However, a too high modulation frequency could cause significant attenuation of the FMM signals as the signal intensity is proportional to $e^{-2 \pi f \tau}$ in which $f$ is the modulation frequency and $\tau$ is the lifetime of fluorescent probes. As most of the fluorescent molecules have a typical lifetime of a few nanoseconds, deterioration of the signal-to-noise ratio will thus be a serious problem at large penetration depths. The $10 \mathrm{MHz}$ we choose falls in the optimal frequency range for fast image acquisition and negligible signal attenuation, and it is compatible with available demodulation components. In future, the issues of inherent Poisson statistical noise that gradually becoming significant in the case of low intensity signal related to deep imaging of biological tissues and further improvement of the image quality with the inclusion of Poisson noise removal will be studied.

\section{Conclusions}

It is a seemingly endless endeavour as microscopists seek to push ever deeper into biological specimens, while resolving micro-structural details and observing specific 
molecular events. This book chapter describes the development of high-speed focal modulation microscopy and the corresponding technological advancement, in which the aim is to extend the penetration depth limit in thick specimens, without the necessity of the usage of long wavelength excitation or non-linear effect, i.e. currently two main approaches for achieving large penetration depth in biological specimens. FMM has thus far proved to be a significant improvement over the conventional confocal microscopy in term of SBR ratio at large penetration depths and potentially useful for cellular and molecular in vivo imaging. Its overall low-cost implementation also means its potential to be widely adopted as a basic research tool in biomedical and clinical applications.

In summary, the principles of FMM is elaborated and two implementations of FMM which varies in term of phase modulation schemes - double-mirrors phase modulator and FMM based on acousto-optical modulator that are significantly progressive in term of stability and image performance as compared to first FMM prototype based on double mirror spatial phase modulator (Chen, Wong et al. 2008) are being demonstrated. It is worth to mention that the modulation frequency is greatly enhanced from $\mathrm{kHz}$ range to $\mathrm{MHz}$ range, and the accompanied benefits include higher image acquisition speed and possible adaptation to fluorescence lifetime imaging. We also demonstrated the capability of FMM in improving the imaging contrast of various types of samples over a large penetration depth. We also highlight that FMM can operate in both fluorescence and back-scattered/reflectance mode. This is contrasted to the non-linear methods such as two-photon microscopy which only improve the imaging depth in fluorescence mode. FMM can switch between fluorescence and back-scattered/reflectance mode by enabling/disabling the phase modulation components in the illumination arm, thus allowing observation of surface topology and tissue morphology based on the back-scattered/reflectance mode as well as rich functional and molecular-specific information through the fluorescence mode.

\subsection{Future directions}

We believe FMM technique will be useful for many biological applications, which large penetration depth is required. Future works to combine the technique of FMM with multiphoton microscopy and microendoscopy are underway to further improve the penetration depth achievable by optical techniques through better rejection of background fluorescence; and for clinical usage to help in early disease detection and diagnosis. On top of that, the application of adaptive optics in FMM to pre-conditioning the beam profile of the excitation beam will further improve the modulation efficiency of FMM, which currently hampered by optical aberrations due to biological samples inhomogeneity. Much stronger FMM signal can be obtained if these optical aberration effects can be dampened which allows better focusing at the focal region and more efficient generation of intensity modulation. The development of FMM microendoscopy, thanks to the technological advancement in miniature fiber-optics and micro-mechanical scanning mechanism, will be invaluable for in vivo cellular-level imaging of tissues in regions that are otherwise inaccessible by bench-top microscope, such as the lung, bladder, cervix, uterus, colon, and peritoneum (Sabharwal, Rouse et al. 1999; Flusberg, Cocker et al. 2005). This ability enables determination of abnormalities localized on the surface or those being more aggressive (i.e. invading below the surface) which will be crucial for early cancer detection.

In addition, the high modulation frequency of FMM based on acousto-optical modulators can also be further developed for fluorescence lifetime imaging. Fluorescence lifetime 
measurements are independent of fluorophores concentrations and thus insensitive to wavelength-dependent light scattering, making it attractive especially in certain scenario such as when uniform illumination for visualization of fluorescent probes deep in tissues cannot be easily achieved over the area of interest owing to its irregular surface (Sun, Phipps et al. 2009).

\subsection{Take home message}

It is always a trade-off between the penetration depths limit and spatial resolutions when adopting an optical microscopy technique in visualization of thick biological specimens. Depending on the applications, it is important to balance the overall cost and imaging qualities to maximize the research outputs. With the advancement of optical engineering, faster scanning mechanisms and higher-performance photodetector, it is expected that biomedical imaging will be benefited with higher-throughput and larger penetration depths.

\section{References}

Axelrod, D. (2003). "Total internal reflection fluorescence microscopy in cell biology." Biophotonics, Pt B 361: 1-33.

Chen, N., C. H. Wong, et al. (2008). "Focal modulation microscopy." Opt Express 16(23): 18764-18769.

Chen, N., C. H. Wong, et al. (2008). "Focal modulation microscopy." Optics Express 16(23): 18764-18769.

Chong, S. P., C. H. Wong, et al. (2010). "Focal modulation microscopy: a theoretical study." Optics letters 35(11): 1804-1806.

Conchello, J. A. and J. W. Lichtman (2005). "Optical sectioning microscopy." Nature methods 2(12): 920-931.

Deng, X. and M. Gu (2003). "Penetration depth of single-, two-, and three-photon fluorescence microscopic imaging through human cortex structures: Monte Carlo simulation." Applied Optics 42(16): 3321-3329.

Denk, W., J. H. Strickler, et al. (1990). "Two-photon laser scanning fluorescence microscopy." Science 248(4951): 73-76.

Detrich, H. W., 3rd, M. Westerfield, et al. (2010). "The zebrafish: cellular and developmental biology, part A. Preface." Methods Cell Biol 100: xiii.

Dittrich, P. S. and P. Schwille (2001). "Photobleaching and stabilization of fluorophores used for single-molecule analysis with one- and two-photon excitation." Applied Physics B-Lasers and Optics 73(8): 829-837.

Dunn, A. K., C. Smithpeter, et al. (1996). "Sources of contrast in confocal reflectance imaging." Applied Optics 35(19): 3441-3446.

Dyba, M. and S. W. Hell (2002). "Focal spots of size lambda/23 open up far-field fluorescence microscopy at $33 \mathrm{~nm}$ axial resolution." Physical review letters 88(16): 163901.

Fercher, A. F. (2010). "Optical coherence tomography - development, principles, applications." Zeitschrift Fur Medizinische Physik 20(4): 251-276.

Fercher, A. F., W. Drexler, et al. (2003). "Optical coherence tomography - principles and applications." Reports on Progress in Physics 66(2): 239-303. 
Firbank, M., M. Oda, et al. (1995). "An improved design for a stable and reproducible phantom material for use in near-infrared spectroscopy and imaging." Physics in Medicine and Biology 40(5): 955-961.

Flusberg, B. A., E. D. Cocker, et al. (2005). "Fiber-optic fluorescence imaging." Nat Methods 2(12): 941-950.

Fujimoto, J. G. (2003). "Optical coherence tomography for ultrahigh resolution in vivo imaging." Nature biotechnology 21(11): 1361-1367.

Fujimoto, J. G., M. E. Brezinski, et al. (1995). "Optical Biopsy and Imaging Using Optical Coherence Tomography." Nature Medicine 1(9): 970-972.

Gan, X., S. Schilders, et al. (1997). "Combination of annular aperture and polarization gating methods for efficient microscopic imaging through a turbid medium: Theoretical analysis." Microscopy and Microanalysis 3(6): 495-503.

$\mathrm{Gu}$, M. (1996). Principles of three dimensional imaging in confocal microscopes. Singapore ; River Edge, NJ, World Scientific.

$\mathrm{Gu}$, M. (2000). Advanced optical imaging theory. Berlin ; New York, Springer.

$\mathrm{Gu}$, M. and C. J. R. Sheppard (1991). "3-Dimensional Imaging in Confocal Fluorescent Microscopy with Annular Lenses." Journal of Modern Optics 38(11): 2247-2263.

Gustafsson, M. G., D. A. Agard, et al. (1999). "I5M: 3D widefield light microscopy with better than $100 \mathrm{~nm}$ axial resolution." Journal of microscopy 195(Pt 1): 10-16.

Hell, S. W., E. H. K. Stelzer, et al. (1994). "Confocal Microscopy with an Increased Detection Aperture - Type-B 4pi Confocal Microscopy." Optics Letters 19(3): 222-224.

Helmchen, F. and W. Denk (2005). "Deep tissue two-photon microscopy." Nature methods 2(12): 932-940.

Huang, D., E. A. Swanson, et al. (1991). "Optical coherence tomography." Science 254(5035): 1178-1181.

Huisken, J., J. Swoger, et al. (2004). "Optical sectioning deep inside live embryos by selective plane illumination microscopy." Science 305(5686): 1007-1009.

Izatt, J. A., M. R. Hee, et al. (1994). "Optical coherence microscopy in scattering media." Optics letters 19(8): 590-592.

Jain, R. K., L. L. Munn, et al. (2002). "Dissecting tumour pathophysiology using intravital microscopy." Nature Reviews Cancer 2(4): 266-276.

Keller, P. J., A. D. Schmidt, et al. (2010). "Fast, high-contrast imaging of animal development with scanned light sheet-based structured-illumination microscopy." Nature methods 7(8): 637-U655.

Keller, P. J., A. D. Schmidt, et al. (2008). "Reconstruction of Zebrafish Early Embryonic Development by Scanned Light Sheet Microscopy." Science 322(5904): 1065-1069.

Keller, P. J. and E. H. Stelzer (2008). "Quantitative in vivo imaging of entire embryos with Digital Scanned Laser Light Sheet Fluorescence Microscopy." Current opinion in neurobiology 18(6): 624-632.

Kempe, M., W. Rudolph, et al. (1996). "Comparative study of confocal and heterodyne microscopy for imaging through scattering media." Journal of the Optical Society of America a-Optics Image Science and Vision 13(1): 46-52.

Kempe, M., A. Thon, et al. (1994). "Resolution Limits of Microscopy through Scattering Layers." Optics Communications 110(5-6): 492-496. 
Kleinfeld, D., P. P. Mitra, et al. (1998). "Fluctuations and stimulus-induced changes in blood flow observed in individual capillaries in layers 2 through 4 of rat neocortex." Proc Natl Acad Sci U S A 95(26): 15741-15746.

Kobat, D., M. E. Durst, et al. (2009). "Deep tissue multiphoton microscopy using longer wavelength excitation." Optics Express 17(16): 13354-13364.

Li, L., R. J. Zemp, et al. (2007). "Photoacoustic imaging of lacZ gene expression in vivo." Journal of Biomedical Optics 12(2): 020504.

Li, M. L., J. T. Oh, et al. (2008). "Simultaneous molecular and hypoxia imaging of brain tumors in vivo using spectroscopic photoacoustic tomography." Proceedings of the Ieee 96(3): 481-489.

Li, Y., Y. Song, et al. (2008). "Direct labeling and visualization of blood vessels with lipophilic carbocyanine dye DiI." Nature protocols 3(11): 1703-1708.

Lunt, S. J., C. Gray, et al. (2010). "Application of intravital microscopy in studies of tumor microcirculation." Journal of Biomedical Optics 15(1): 011113.

Minsky, M. (1961). Microscopy Apparatus. US.

Minsky, M. (1988). "Memoir on Inventing the Confocal Scanning Microscope." Scanning 10(4): 128-138.

Misgeld, T. and M. Kerschensteiner (2006). "In vivo imaging of the diseased nervous system." Nature reviews. Neuroscience 7(6): 449-463.

Mo, W. and N. Chen (2008). "Fast time-domain diffuse optical tomography using pseudorandom bit sequences." Opt. Express 16(18): 13643-13650.

Oheim, M., E. Beaurepaire, et al. (2001). "Two-photon microscopy in brain tissue: parameters influencing the imaging depth." J Neurosci Methods 111(1): 29-37.

Patterson, G. H. and D. W. Piston (2000). "Photobleaching in two-photon excitation microscopy." Biophysical journal 78(4): 2159-2162.

Pavlova, I., M. Williams, et al. (2008). "Understanding the biological basis of autofluorescence imaging for oral cancer detection: high-resolution fluorescence microscopy in viable tissue." Clinical cancer research : an official journal of the American Association for Cancer Research 14(8): 2396-2404.

Pawley, J. B. (2006). Handbook of biological confocal microscopy. New York, NY, Springer.

Prati, F., E. Regar, et al. (2010). "Expert review document on methodology, terminology, and clinical applications of optical coherence tomography: physical principles, methodology of image acquisition, and clinical application for assessment of coronary arteries and atherosclerosis." European Heart Journal 31(4): 401-415.

Razansky, D., M. Distel, et al. (2009). "Multispectral opto-acoustic tomography of deepseated fluorescent proteins in vivo." Nat Photon 3(7): 412-417.

Sabharwal, Y. S., A. R. Rouse, et al. (1999). "Slit-scanning confocal microendoscope for highresolution in vivo imaging." Applied Optics 38(34): 7133-7144.

Sandison, D. R., D. W. Piston, et al. (1995). "Quantitative Comparison of Background Rejection, Signal-to-Noise Ratio, and Resolution in Confocal and Full-Field LaserScanning Microscopes." Applied Optics 34(19): 3576-3588.

Schmitt, J. M., A. Knuttel, et al. (1994). "Confocal Microscopy in Turbid Media." Journal of the Optical Society of America a-Optics Image Science and Vision 11(8): 22262235. 
Schmitt, J. M., A. Knuttel, et al. (1994). "Confocal microscopy in turbid media." Journal of the Optical Society of America. A, Optics, image science, and vision 11(8): 22262235.

Sheppard, C. J. R. and M. Gu (1991). "3-Dimensional Optical Transfer-Function for an Annular Lens." Optics Communications 81(5): 276-280.

Sheppard, C. J. R. and T. Wilson (1980). "Image-Formation in Confocal Scanning Microscopes." Optik 55(4): 331-342.

Smithpeter, C. L., A. K. Dunn, et al. (1998). "Penetration depth limits of in vivo confocal reflectance imaging." Applied Optics 37(13): 2749-2754.

Sun, Y., J. Phipps, et al. (2009). "Fluorescence lifetime imaging microscopy: in vivo application to diagnosis of oral carcinoma." Opt Lett 34(13): 2081-2083.

Svoboda, K., F. Helmchen, et al. (1999). "Spread of dendritic excitation in layer 2/3 pyramidal neurons in rat barrel cortex in vivo." Nat Neurosci 2(1): 65-73.

Svoboda, K. and R. Yasuda (2006). "Principles of two-photon excitation microscopy and its applications to neuroscience." Neuron 50(6): 823-839.

Theer, P. and W. Denk (2006). "On the fundamental imaging-depth limit in two-photon microscopy." Journal of the Optical Society of America. A, Optics, image science, and vision 23(12): 3139-3149.

Theer, P., M. T. Hasan, et al. (2003). "Two-photon imaging to a depth of 1000 microm in living brains by use of a Ti:Al2O3 regenerative amplifier." Opt Lett 28(12): 10221024.

Theer, P., M. T. Hasan, et al. (2003). "Two-photon imaging to a depth of $1000 \mathrm{mu} \mathrm{m}$ in living brains by use of a Ti : Al2O3 regenerative amplifier." Optics Letters 28(12): 10221024.

Vakoc, B. J., R. M. Lanning, et al. (2009). "Three-dimensional microscopy of the tumor microenvironment in vivo using optical frequency domain imaging." Nat Med 15(10): 1219-1223.

Verveer, P. J., J. Swoger, et al. (2007). "High-resolution three-dimensional imaging of large specimens with light sheet-based microscopy." Nature methods 4(4): 311-313.

Wang, L. V. (2009). "Multiscale photoacoustic microscopy and computed tomography." Nature photonics 3(9): 503-509.

Wang, X., Y. Pang, et al. (2003). "Noninvasive laser-induced photoacoustic tomography for structural and functional in vivo imaging of the brain." Nature biotechnology 21(7): 803-806.

Wang, X., Y. Xu, et al. (2002). "Photoacoustic tomography of biological tissues with high cross-section resolution: reconstruction and experiment." Medical Physics 29(12): 2799-2805.

Wang, X. D., G. Ku, et al. (2004). "Non-invasive functional photoacoustic tomography of blood oxygen saturation in the brain." Photons Plus Ultrasound: Imaging and Sensing 5320: 69-76.

Wong, C. H., S. P. Chong, et al. (2009). "Simple spatial phase modulator for focal modulation microscopy." Applied Optics 48(17): 3237-3242.

Yang, S., D. Xing, et al. (2007). "Functional imaging of cerebrovascular activities in small animals using high-resolution photoacoustic tomography." Medical Physics 34(8): 3294-3301. 
Yao, D. K., K. Maslov, et al. (2010). "In vivo label-free photoacoustic microscopy of cell nuclei by excitation of DNA and RNA." Opt Lett 35(24): 4139-4141.

Ying, J., F. Liu, et al. (1999). "Spatial distribution of two-photon-excited fluorescence in scattering media." Appl Opt 38(1): 224-229.

Zeng, C., S. Vangveravong, et al. (2007). "Subcellular localization of sigma-2 receptors in breast cancer cells using two-photon and confocal microscopy." Cancer research 67(14): 6708-6716. 


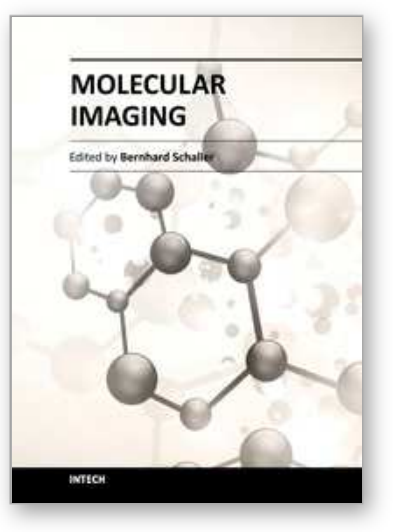

\author{
Molecular Imaging \\ Edited by Prof. Bernhard Schaller
}

ISBN 978-953-51-0359-2

Hard cover, 390 pages

Publisher InTech

Published online 16, March, 2012

Published in print edition March, 2012

The present book gives an exceptional overview of molecular imaging. Practical approach represents the red thread through the whole book, covering at the same time detailed background information that goes very deep into molecular as well as cellular level. Ideas how molecular imaging will develop in the near future present a special delicacy. This should be of special interest as the contributors are members of leading research groups from all over the world.

\title{
How to reference
}

In order to correctly reference this scholarly work, feel free to copy and paste the following:

Nanguang Chen, Guangjun Gao and Shau Poh Chong (2012). Focal Modulation Microscopy: Principle and Techniques, Molecular Imaging, Prof. Bernhard Schaller (Ed.), ISBN: 978-953-51-0359-2, InTech, Available from: http://www.intechopen.com/books/molecular-imaging/focal-modulation-microscopy-theory-and-technique

\section{INTECH}

open science | open minds

\section{InTech Europe}

University Campus STeP Ri

Slavka Krautzeka 83/A

51000 Rijeka, Croatia

Phone: +385 (51) 770447

Fax: +385 (51) 686166

www.intechopen.com

\section{InTech China}

Unit 405, Office Block, Hotel Equatorial Shanghai

No.65, Yan An Road (West), Shanghai, 200040, China

中国上海市延安西路65号上海国际贵都大饭店办公楼405单元

Phone: +86-21-62489820

Fax: +86-21-62489821 
(C) 2012 The Author(s). Licensee IntechOpen. This is an open access article distributed under the terms of the Creative Commons Attribution 3.0 License, which permits unrestricted use, distribution, and reproduction in any medium, provided the original work is properly cited. 